\title{
Aristotelische Biologie. Eine Synopsis
}

D O I: $10.14746 / p e a .2020 .1 .4$

MARTIN F. MEYER / Universität Koblenz/

Aristoteles wurde in der Tradition fast exklusiv als Philosoph wahrgenommen. Er selbst hätte sich wohl eher als Naturwissenschaftler (als pvoıkós) gesehen, vornehmlich als Biologe und zoologischer Forscher (Kullmann 2014). Ein Blick in das Corpus Aristotelicum, das Konvolut der unter seinem Namen tradierten Schriften, bestätigt das. Gliedert man die Schriften nach Themengruppen und den auf sie entfallenden Seiten, so ergibt sich folgendes Bild: 


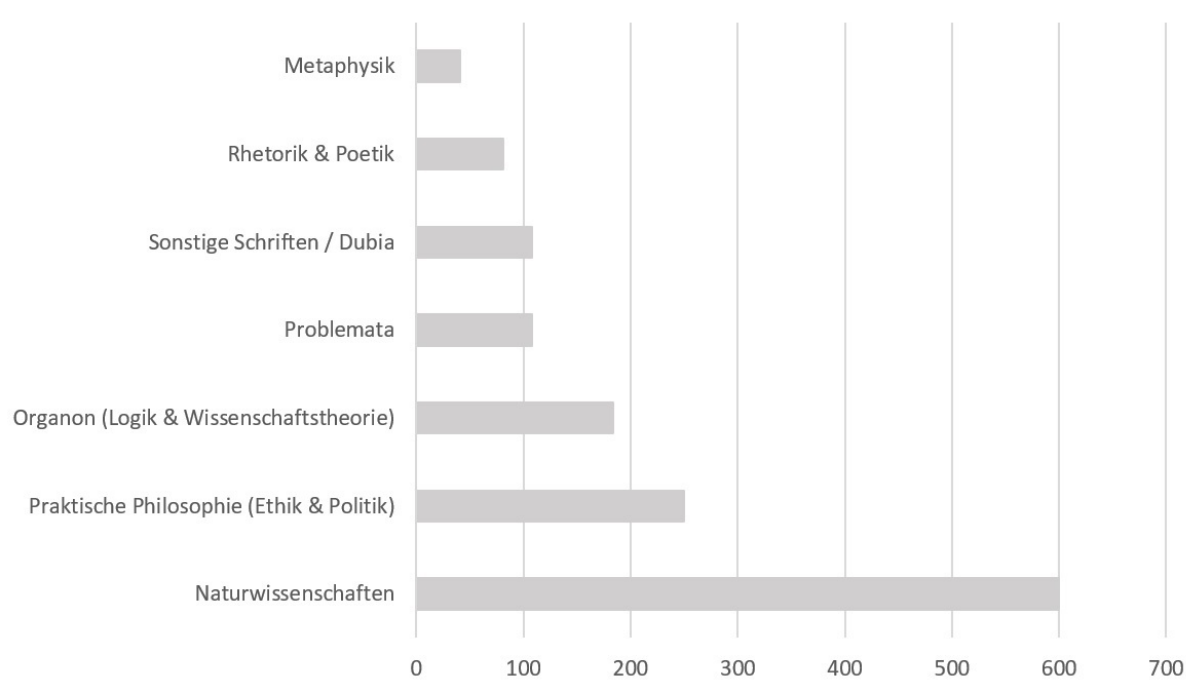

Schriftengruppen im Corpus Aristotelicum (1462 Bekker-Seiten) nach Umfang

Ein Großteil der Werke (mehr als 4o Prozent) behandelt naturwissenschaftliche Fragen. Die naturwissenschaftlichen Schriften ihrerseits widmen sich primär biologischen und hier ganz überwiegend (zu mehr als 95 Prozent) zoologischen Themen. Aristoteles erwähnt in diesen Schriften gut 550 Tier- und 6o Pflanzenarten. Er begann die biologischen Forschungen nach seiner Akademiezeit im Alter von 37 Jahren als er sich zwischen 347 und 335 v. Chr. im nordost-ägäischen Raum aufhielt. Er lebte in Assos (in der Troas) und auf der gegenüberliegenden Insel Lesbos, wo er Theophrast (den »Vater der Botanik«) zur Mitarbeit an den Forschungen bewegen konnte. Die Troas war seit Homer als Durchzugsgebiet von Zugvögeln wie Kranichen bekannt (Kraak 1940). Die Ilias kennt das »quellenreiche« Idagebirge als »Mutter der Tiere« (Il. VIII 47; XIV 283; XV 151). Im Altertum lebten dort noch Löwen und Panther. Der Aufenthalt in diesen auch an maritimer Fauna reichen Arealen (wie der Lagune von Phyrra) erklärt, warum die aristotelische Zoologie auffallend viele Auskünfte zu Fischen und Meerestieren enthält. ${ }^{1}$ Für die Forschung sammelte Aristoteles alle Informationen, die er bekommen konnte. Er befragte Bauern, Fischer und Imker zu Tieren und Pflanzen. Hinsichtlich der exotischen Fauna in Afrika und Asien ließ er sich von Reiseberichten - etwa aus Herodots Historien - inspirieren. Die Quellen waren nicht immer zuverlässig. Aristoteles hat die Zeugnisse, wo er konnte, überprüft und korrigiert. ${ }^{2}$ Wolfgang Kullmann hat gezeigt, daß Aristoteles

${ }^{1}$ Vgl. Leroi (2017: 25-26). Als der schottische Gelehrte D‘Arcy Wentworth Thompson (1910) die Historia animalium (Aristoteles' zoologisches Hauptwerk) ins Englische übersetzte, bemerkte er, daß Aristoteles einen Großteil seiner meeresbiologischen Daten in der Lagune von Phyrra auf Lesbos gesammelt hatte.

${ }^{2}$ Vgl. Kullmann (2014); Leroi (2017). Kullmann (2014 - Kap. 6) demonstriert, daß Aristoteles die zoologischen Quellen akribisch geprüft hat. So ist in seinen Schriften an 25 Stellen vom Krokodil die Rede. Schon in der Renaissance wußte man, daß die meisten Angaben auf Herodot zurückgehen. Im Rekurs auf eine frühere Studie (Kullmann 2000) zeigt Kullmann, wie Aristoteles die Angaben der Historien kritisch geprüft hat. Seine 
ähnlich wie Theophrast weite Forschungsreisen unternommen hat (nach Kullmann ist er bis auf die Krim gereist). ${ }^{3}$ Aristoteles schildert die innere Anatomie von ca. 110 Tieren; er sezierte mehr als 60 Spezies. Die Sektionen fanden ihren Niederschlag in einem voluminösen Anatomischen Atlas - ein Bildwerk mit Zeichnungen zu den inneren und äußeren Organen der Tiere, das er in den Vorlesungen am Lykeion nutzte (Stückelberger 1998: 287-307). Auf den Atlas verweist er an 26 Stellen in den zoologischen Schriften. Seinen Hörern rät er, das, was in den Kursen logisch erschlossen werde, in den »Anatomai« direkt anschauen $(\theta \varepsilon \omega \rho \varepsilon \tilde{v}) .{ }^{4}$ Der Anatomische Atlas ist seit der Spätantike verloren. David Koutsogiannopoulos und die Papyrologin Grace Ioannidou haben jüngst versucht, einige Zeichnungen zu rekonstruieren (Leroi 2017: 508-510).

Große Fachgelehrte würdigten den Biologen Aristoteles mit hoher Anerkennung. Zu den prominesten Stimmen gehört neben D‘Arcy Wentworth Thompson und Ernst Mayr auch Charles Darwin, der im Februar 1882 notiert, gegen Aristoteles seien seine eigenen Vorbilder Cuvier und Linné »nur bloße Schuljungs «. In der europäischen Wissenschaftstradition war der aristotelischen Biologie lange ein Mauerblümchensein beschieden. Die hellenistischen und römischen Denker kannten die zoologischen Schriften nicht. In der mittelalterlichen Aristoteles-Rezeption spielte sein biologisches Oeuvre kaum eine Rolle. Es gab Ausnahmen wie Albertus Magnus - und auch die Schriften der Parva naturalia fanden unter Medizinern etwas Aufmerksamkeit. Erst seit der Renaissance wurden die zoologischen Schriften von einem breiteren Fachpublikum diskutiert. Ein gutes Beispiel dafür sind die in fünf Bänden von 1551 bis 1587 erschienenen Bände der Historia animalium von Conrad Gessner. Die folgenden Zeilen geben eine Synopsis zu Anliegen, Methode, Systematik und Erklärungen der aristotelischen Biologie.

\section{Epistemische Motive}

Zu Aristoteles' Zeit war die wissenschaftliche Beschäftigung mit Tieren und Pflanzen nicht selbstverständlich. Zwar gab es vorher schon wichtige Beobachtungen zu Flora und Fauna. Von einer systematischen Erforschung des Lebens konnte jedoch keine Rede sein. Eine Ausnahme war Demokrit. Nach Aristoteles' Zeugnis (PA I 1, 642b1-2) steht

anatomischen Beschreibungen sprechen dafür, daß er zwar kein lebendes Krokodil gesehen hat, aber tote Krokodile seziert haben könnte (Kullmann 2000: 113-131). Kullmann (2000: 131-134) zeigt an den Pragmatien zu Elefanten, zu Strauß, Rhinozeros, Kamel und Flußpferd, daß Aristoteles auch die diesbezüglichen Informationen Herodots nachgeprüft hat.

${ }^{3}$ Vgl. Kullmann (2014: 78-112). Kullmann zeigt in Anlehnung an die Theophrast-Studien von Maxwell Stuart (1996), daß Aristoteles diese Reisen teils gemeinsam mit Theophrast unternommen hat. Eine Vielzahl zoologischer Textstellen belegt, daß Aristoteles in den 13 Jahren nach seiner Akademiezeit u.a. in die Troas, nach Lesbos und bis zur Krim gereist ist. Wissenschaftliche Interessen waren das zentrale Motiv der Reisen (Kullmannn 2014: 80-81: geographische Karten zu den Reisen). Kullmann unterlegt die Ausführungen mit Parallelstellen aus Theophrasts botanischen Werken. Es sei wahrscheinlich, daß Aristoteles und Theophrast arbeitsteilig geforscht hätten.

${ }^{4}$ Vgl. Arist. Resp. 16, 478a26-28 und 478a34-b27. 


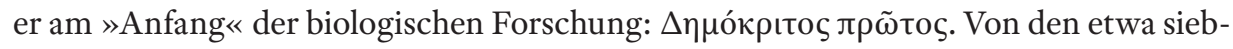
zig Schriften, die Demokrit verfaßt haben soll, ist keine einzige erhalten. Sein biologisches Denken läßt sich nur umrißhaft rekonstruieren (Meyer 2009). Generell stand die Beschäftigung mit Käfern, Spinnen oder Muscheln nicht im Fokus jener freien Männer der Antike, die politische Karrieren anstrebten oder sich in Epistemen wie Geometrie, Arithmetik oder Astronomie engagierten. Biologische Fragen wurden gar verspottet. ${ }^{5}$ Aristoteles verteidigt sein Forschungsvorhaben daher in einer großen Werberede. Obwohl er Astronomie und Biologie für »gleich reizvoll« hält, plädiert er für das intensive Studium des Lebendigen:

Wie es sich nun aber so trifft, besitzen wir über jene [Gegenstände], die ehrwürdig und göttlich sind, weniger Einsichten (denn über die Ausgangspunkte, von denen aus wir jene erforschen können, und über die Dinge, die wir zu wissen begehren, ist nur außerordentlich wenig

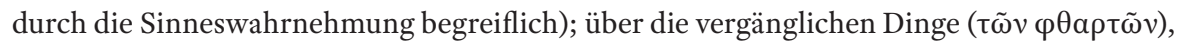
Pflanzen sowohl als auch Tiere sind unsere Kenntnisse weit reichlicher, da wir ja zusammen mit ihnen aufwachsen ( $P A$ I 5, 644b 24-29) [ ̈Ü. angelehnt an Gigon].

Dies nur ein kleiner Ausschnitt des Plädoyers (Kullmann 1997: 43-62). Leicht vergröbert lassen sich drei Leitmotive für das biologische Erkenntnisinteresse anführen:

a. Erstens sind alle natürlichen Dinge gleichermaßen der wissenschaftichen Erforschung wert, sofern sie der Naturerkenntnis dienen. Die Untersuchung der nur scheinbar unwürdigeren Entitäten der Biologie verspricht, wenn man es richtig anstellt, keinen geringeren Erkenntnisgewinn als etwa astronomisches Wissen.

b. Zweitens verspricht die biologische Forschung wegen der besseren (in unserer Vertrautheit mit irdischen Dingen begründeten) Beobachtungsoptionen mehr gesichertes Wissen und hinsichtlich der Naturerkenntnis höhere Wissenserträge. In der Astronomie zeigt sich die natürliche Regelhaftigkeit (»Ordnung und Bestimmtheit«) zwar deutlicher. Wegen unserer limitierten Beobachtungsmöglichkeiten ist sicheres Wissen hier aber schwerer zu erlangen. In der Biologie sind die Regularitäten zwar undeutlicher. Dieses Defizit wird also durch das reichere empirische Datenmaterial kompensiert.

5 Vgl. Düring (1966: 525-526). Beispielhaft ist, wie sich der Komiker Epikrates über umständliche Versuche der Akademiker mokiert, das Wesen eines Kürbisses zu bestimmen: »A. Was tun jetzt Platon, Speusipp und Menedemos? - B. Darüber weiß ich Bescheid. Beim Panathenäerfest sah ich eine Schar Jünglinge in der Akademie und hörte ein seltsames Gespräch. Offenbar beschäftigten sie sich damit, Tiere, Bäume und Pflanzen in Arten und Gattungen einzuteilen; sie waren eben dabei zu bestimmen, zu welcher Gattung ein Kürbis gehört. - A. Gelang es ihnen? - B. Zuerst waren alle stumm und nachdenklich und grübelten mit gesenkten Häuptern. Da kam einer der Jünglinge plötzlich mit einer Definition heraus: »Eine Gartenpflanze mit sphärischer Frucht «. Ein anderer sagte, der Kürbis gehöre zur Gattung >Bäume<. Ein Arzt aus Sizilien, der dieses Geschwätz hörte, lachte roh über ihre Einfalt. - A. Waren sie nicht empört? Denn so darf man sich ja doch nicht in der Akademie benehmen. - B. Nein, die Jünglinge nahmen davon keine Notiz. Doch, jetzt ergriff Platon das Wort. Ruhig und gelassen begann - er von Grund auf ihnen zu erklären, zu welcher Gattung der Kürbis gehört, und als ich sie verließ, waren sie immer noch mit dem Klassifizieren beschäftigt «. 
c. Drittens verspricht die Biologie bedeutende Einsichten über den Menschen. Aristoteles begründet das v.a. damit, daß wir Menschen aus denselben Teilen bestehen wie die Tiere (ein Argument, das schon in einigen Frühschriften des Corpus Hippocraticum begegnet; vgl. Oser-Grote 2004).

Aristoteles grenzt die Biologie nach zwei Richtungen hin ab: Einerseits gegen die Medizin, andererseits gegen die praktische Philosophie. Anders als in der Biologie gelte das medizinische Interesse fast exklusiv dem menschlichen Körper. Aristoteles, der selbst aus einer beachtlichen Ärztedynastie stammte, schreibt, das Ziel der ärztlichen Kunst sei die Heilung. Allgemeine Naturerkenntnis sei in der Medizin eher Mittel als Zweck. ${ }^{6}$ Nur im Idealfall seien gute Ärzte auch gute Naturwissenschaftler. Umgekehrt gelte:

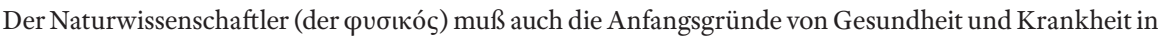
seine Betrachtung einbeziehen, denn weder Gesundheit noch Krankheit kann leblosen Wesen zukommen. Daher werden auch die meisten Naturphilosophen letztlich zu medizinischen Fragen geführt -

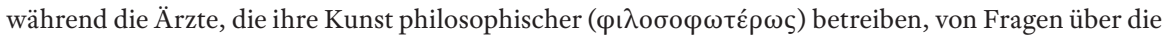

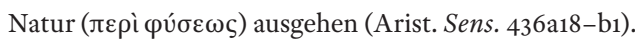

Die Heilung richte sich stets auf das einzelne Individuum. Die biologische Erklärung aber gelte den Spezies (van der Eijk 2005). Die Grenze zur praktischen Philosophie (zu Politik und Ethik) sieht er darin, daß die Biologie auf die Erklärung von Leben zielt, die praktische Philosophie auf Bedingungen für das gute Leben ( $\varepsilon \tilde{u} \zeta \tilde{\eta} v)$. Die Ethik ist eher eine Wissenschaft »des Dass «, des tò ötı (EN I 7, 1098b1-4). Sie unterscheidet sich methodisch von der Biologie, die (wie alle Naturwissenschaften) auf Ursachenerklärung zielt. Aristoteles' Methode ist ein bottom-up-top-down-Verfahren. Als Empiriker sammelt er relevante Fakten, um via Induktion allgemeine Prämissen zu etablieren, aus denen sich deduktive Erklärungen. Kullmann hat klargestellt, daß Aristoteles zwei Typen von Erklärungen anvisiert: (a) Erklärungen des »Dass« ( faktisch der Fall ist (z.B. ob/dass bestimmte Tiere atmen oder nicht); (b) Erklärungen

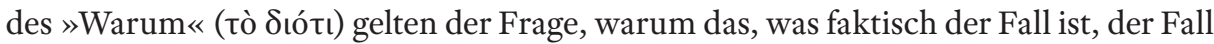
ist (z.B. warum bestimmte Tiere atmen). Erst mit der kausalen Erklärung eines Sachverhalts gelangt die naturwissenschaftliche Explanation ans Ziel.

${ }^{6}$ Vgl. Arist. Resp. 11, 480b22-30: »Sich über die Ursachen von Gesundheit und Krankheit zu äußern, ist nicht allein Angelegenheit des Arztes, sondern bis zu einem bestimmten Grad auch Sache des Naturforschers ( den und inwieweit es sich um verschiedene Formalobjekte handelt, darf man nicht übersehen, wenn auch die Erfahrung bezeugt, daß beider Arbeit bis zu einem gewissen Punkte parallel geht. Denn alle Ärzte, die Niveau

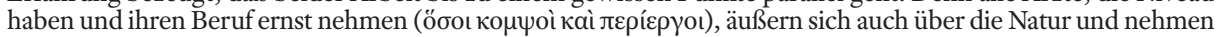

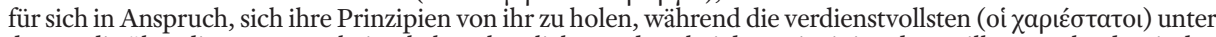
denen, die über die Natur gearbeitet haben, letztlich geradezu bei den Prinzipien der Heilkunst gelandet sind «; vgl. Meyer (2015a: 268-266). 


\section{Naturwissenschaftlicher Kontext}

Für Aristoteles ist die Biologie eine Naturwissenschaft. Er entwickelte als erster Denker ein distinktes Konzept von Naturwissenschaft. Dies ist der Ertrag seiner Physikvorlesungen, die man mißversteht, wenn man hier konkrete Erklärungen wie in der neuzeitlichen Physik erwartet. Die Objekte der aristotelischen Physik sind »natürliche Körper «. Natürliche Körper unterscheiden sich von nichtnatürlichen Körpern (Artefakten) darin, daß sie das »Prinzip ihrer Bewegung in sich « tragen. Somit stehen Fragen nach der Bewegung im Zentrum der Naturwissenschaft. Aristoteles faßt den Term »Bewegung «

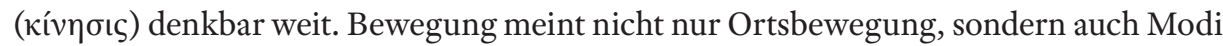
der Veränderung wie Wachstum oder Vergehen. Ziel der Naturwissenschaft ist es, die »Physis« (die Formbestimmtheit) einer natürlichen Sache kausal zu erklären. Erklären meint hier zu erklären, »was-es-macht«, daß eine natürliche Sache so ist wie sie ist. Im Fall der Artefakte würde man diese Frage, wie Aristoteles betont, so angehen: Fragt man, was ein Haus zu diesem Haus macht, so würde man (a) die Funktion des Hauses nennen, (b) die Form (den Plan des Architekten), (c) das Material, aus dem es besteht (Ziegelsteine, Holz) und (d) die Wirkursache (die Handwerker). Es ist heute üblich, diese vier Ursachentypen mit den lateinischen Worten als causa finalis, causa formalis, causa materialis und causa efficiens zu bezeichnen. Es ist unwahrscheinlich, daß Aristoteles das Vier-Ursachen-Modell auf unbelebte Körper anwenden wollte. Die frühneuzeitlichen Denker haben Aristoteles vorgeworfen, er habe Zielursachen in der unbelebten Natur angenommen. In der Forschung hat sich seit Ende der 196oer Jahre die Einsicht durchgesetzt, daß es bei Aristoteles keine sogenannte »Universalteleologie « gibt. Um diesem Mißverständnis vorzubeugen, spricht man mit Blick auf die aristotelische Biologie (in Anlehnung an moderne Biologen) besser von »teleonomischen Prozessen «. Beispielsweise läßt sich das Wachstum eines Affen als »teleonomischer Prozeß « beschreiben: Ein Affe wächst nicht unbegrenzt. Durch Querverweise in den Schriften ist belegt, daß Aristoteles die naturwissenschaftlichen Forschungen nach der Art eines systematischen Curriculums am Lykeion vorgetragen hat. Wie in anderen von ihm behandelten Wissenschaften beginnt er auch in der Physikvorlesung mit prinzipiellen Überlegungen, um vorab das Forschungsfeld und die passenden Methoden zu skizzieren. Erst im Anschluß an diese vorgelagerte Prinzipienforschung beginnt der Weg der Erklärung - zuerst die Erklärung der unbelebten Natur - dann die Erklärung der komplexeren Gegenstände der belebten Welt. Der Plan des aristotelischen Curriculums läßt sich anhand der erhalten naturwissenschaftlichen Schriften verdeutlichen.

7 Vgl. Arist. Ph. II 1, 192b8-14: »Von den realen Dingen ( $\tau \tilde{\omega} \nu$ oै $v \tau \omega v)$ existieren die einen von Natur aus

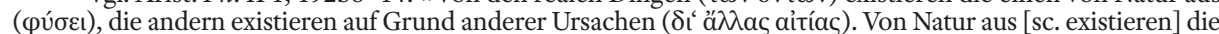

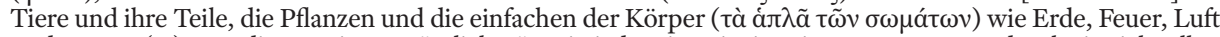
und Wasser (...). Von diesen Dingen nämlich trägt ein jedes ein Prinzip seiner Bewegung und Ruhe in sich selbst

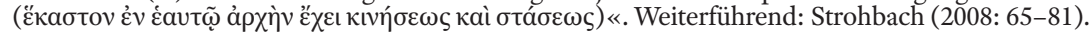




\begin{tabular}{|c|c|}
\hline Physica & $\begin{array}{l}\text { Allgemeine Bestimmung der Naturwissenschaft } \\
\text { und ihrer Grundbegriffe }\end{array}$ \\
\hline De generatione et corruptione & $\begin{array}{l}\text { Zusätzliche Reflexionen über Werden und Verge- } \\
\text { hen }\end{array}$ \\
\hline De caelo & $\begin{array}{l}\text { Astrophysische Erklärungen über die Himmelsphä- } \\
\text { nomene }\end{array}$ \\
\hline Meteorologica & Über die Dinge unterhalb des Mondes \\
\hline De anima $\mathrm{I}-\mathrm{II} 5$ & $\begin{array}{l}\text { Allgemeine Bestimmung der Biologie und ihrer } \\
\text { Grundbegriffe }\end{array}$ \\
\hline De plantis & $\begin{array}{l}\text { Grundbegriffe der Botanik, allgemeine botanische } \\
\text { Erklärungen }\end{array}$ \\
\hline Historia animalium I-IV 7 & Vergleichende Anatomie (Erklärung des Dass) \\
\hline De partibus animalium II-IV & Vergleichende Anatomie (Erklärung des Warum) \\
\hline De anima II 5-III, De sensu et sensibilibus & Erklärung der Sinneswahrnehmungen \\
\hline De memoria et reminiscentia & Über Erinnerung und Wiedererinnerung \\
\hline $\begin{array}{l}\text { De somno et vigilia / De insomniis / De divinatione } \\
\text { per somnum }\end{array}$ & Über Wachen und Schlafen \\
\hline De motu animalium / De incessu animalium & Über die Fortbewegung und Bewegung der Tiere \\
\hline Historia animalium $\mathrm{V}-\mathrm{VII}$ & $\begin{array}{l}\text { Über Fortpflanzung und Entwicklung (Erklärung } \\
\text { des Dass) }\end{array}$ \\
\hline De generatione animalium & $\begin{array}{l}\text { Über Fortpflanzung und Entwicklung (Erklärung } \\
\text { des Warum) }\end{array}$ \\
\hline Historia animalium VIII-IX & Über Verhalten und Intelligenz der Tiere \\
\hline De respiratione & Über die Funktion der Atmung \\
\hline De longaevitate & $\begin{array}{l}\text { Über die unterschiedliche Lebensdauer der } \\
\text { Lebewesen }\end{array}$ \\
\hline De juventute et senectute / De vita et morte & Allgemeine Erklärungen über Leben und Tod \\
\hline
\end{tabular}

\section{Prinzipienforschung}

Auch in der Biologie lagert Aristoteles der eigentlichen Erklärung eine Prinzipienforschung vor. Dies hat den Zweck, (a) die hier spezifischen Forschungsgegenstände von Gegenständen anderer Wissenschaften zu unterscheiden und (b) hier passende Methoden vorzuzeichnen. Das Hauptwerk dieser Prinzipienforschung ist die Schrift Über die Psyche (De anima). Wichtige Überlegungen zur Methodik (insb. zur funktionalen Anatomie) finden sich im I. Buch der Schrift De partibus animalium (Kullmann 2007; Meyer 2015: 259-267). Die Schrift De anima hat mit moderner Psychologie nichts gemein. Es geht ihr nicht um mentale Zustände oder individualpsychische Dispositionen. Der Begriff $\cup \chi \eta ́$ hat in der aristotelischen Biologie zwei Funktionen: Die Psyche ist Prinzip (ả $\chi \eta \dot{)})$ 
und Ursache (aitía). Erstens dient die »Psyche« zur Abgrenzung belebter Naturkörper (Pflanzen, Tiere, Menschen) von unbelebten Naturkörpern (Planeten, Sterne, Steine). Der Begriff $\psi v \chi \eta ́$ grenzt die Biologie von jenen Naturwissenschaften ab, die es (wie die Astronomie) mit unbelebten Dingen zu tun haben. Insofern bestimmt der Psyche-Begriff den Objektbereich der Biologie. Um zu verstehen, was er mit $\psi v \chi \eta ́$ meint, ist der begriffsgeschichtliche Hinweis nötig, daß die Griechen lange kein Wort für »Lebewesen « hatten

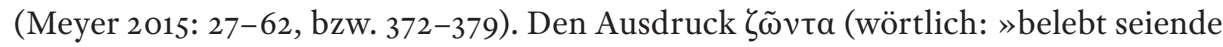
Dinge «) hat Aristoteles erfunden. Als Lebewesen galten seit Pythagoras Entitäten, in denen Psyche ist. Die Vorgänger nannten sie عُ $\mu \psi v \chi \alpha$. (Die verwickelte Geschichte des Psyche-Begriffs kann hier nicht ausgebreitet werden (Meyer 2008; Meyer 2021). Kurz:

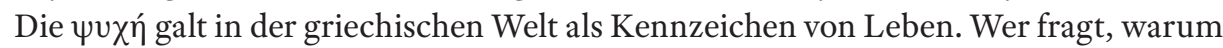
dies so war, sollte Fragen stellen wie: »Warum bewegt sich ein Löwe?« - »Warum wächst eine Rose?«. Die Antworten lauten dahin, daß es etwas gibt, was diese Dinge von selbst (aus sich selbst heraus), »autopoetisch « bewegt. Anders als ein Stein, der nur durch äußere Impulse bewegt wird, entsteht die Bewegung von Rosen oder Delphinen gleichsam von innen. Was nun könnte dieses innere Bewegungsprinzip sein? Die Fragen weisen schon auf die $\psi v \chi \eta \dot{~}$. Aristoteles macht auf den Unterschied von lebenden und unbelebten Dingen aufmerksam, wenn er sagt, eine tote Hand sei strenggenommen gar keine Hand oder allenfalls dem Namen nach eine Hand. ${ }^{8}$ Er begreift das Leben als jene Eigenschaft von funktional organisierter Materie, die diese Materie auf ein bestimmtes Ziel hin so organisiert, daß diese Entität ihrer Form nach genau das ist, was sie ist - und zwar so, daß die Erklärung dieser finalen Organisiertheit (»Teleonomie«) keine andere sein kann als eine Bestimmung des artspezifischen Lebens dieses Wesens. Materie ist für Aristoteles stets formbestimmt. »Materie an sich « wäre keiner wissenschaftlichen Erklärung zugänglich und ein bloß leeres Abstraktum. Jedes konkrete Naturding ist immer schon formbestimmt: Ein Salzblock ist etwas anders als ein Granitfels. Ein Mensch ist etwas anderes als ein Löwe. Ein Knochen etwas anderes als eine Hand. An diesem Punkt kommt die Psyche ins Spiel. Wenn Aristoteles sagt, die Psyche sei ein Prinzip (ả $\chi \eta ́$ ), meint er, daß die Psyche ein Prinzip der Bewegung eines Lebewesens ist. Dies indes ist nur die »allge-

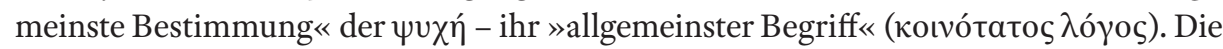
berühmte Definition dieses allgemeinsten Begriffs« lautet:

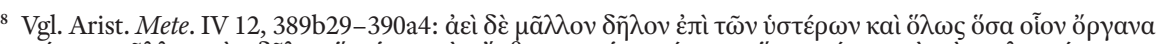

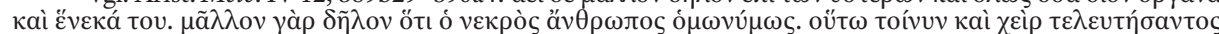

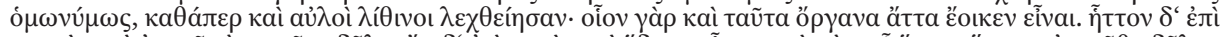

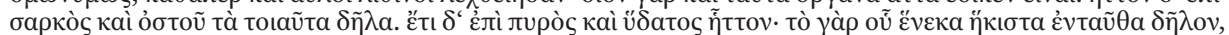

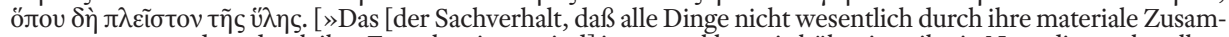
mensetzung, sondern durch ihre Form bestimmt sind] ist um so klarer, je höher jeweils ein Naturding steht; allgemein gesagt, es ist überall deutlich, wo etwas Werkzeug [Organ] ist und einem Zweck dient. So ist es klar genug, daß ein toter Mensch nur dem Namen nach [ein Mensch] ist: So ist die Hand eines Toten nur dem Namen nach [eine Hand], im gleichen Sinne wie auch eine in Stein gebildete Flöte so heißen könnte, auch sie ist ja gewissermaßen wie ein Werkzeug (oíov öpyava). Bei Fleisch und Knochen ist dies weniger deutlich, noch weniger bei Feuer und Wasser, der Zweck ist nämlich da am wenigsten offenbar, wo am meisten Materie ist «]. 
Notwendig also muß die Psyche Wesen (ov̉oía) sein so wie die Form (das عĩooৎ) eines natürlichen Körpers, der seiner Möglichkeit nach Leben besitzt. Das Wesen (die oủoía) aber ist Vollendung (Aktualität) (de An. II 1, 412a19-21).

Die Psyche ist demnach:

a. Ousia im Sinne des Wesens. Beispiel: Es ist das Wesen eines Löwen lebendig zu sein. Ein toter Löwe ist gar kein Löwe (oder allenfalls dem Namen nach).

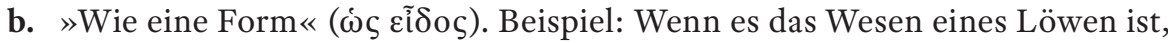
kein Fuchs zu sein, so liegt der Grund dafür, daß er ein Löwe ist und nichts anderes, in seiner Lebendigkeit. Der Grund dafür, daß ein Löwe ein Löwe ist, liegt in der Artform. In der Artform (Spezies) liegt der Grund für seine spezifische Form der Lebendigkeit. Für Aristoteles ist die spezifische Form eines jeden Lebewesens die Artform. Die Spezies macht es, daß ein Löwe ein Löwe oder ein Fuchs ein Fuchs ist. Dies läßt schon ahnen, daß die »Artform « das eigentliche Explanandum der aristotelischen Biologie ist. ${ }^{9}$ Wenn wir wissen wollen, was die Natur (die Physis) eines Löwen ist, müssen wir wissen, was diese besondere Art Panthera leo ausmacht: Wieviel Beine Löwen haben, ob sie ein Gehirn oder Nerven haben, ob sie wachen und schlafen, wie sie sich fortpflanzen usw.

c. Die (erste) Entelechie eines potentiell belebten Körpers. Dies ist die schwierigste Klausel. Aristoteles hat gesehen, daß Individuen in sehr verschiedenen Zuständen existieren; Zustände wie Jugend, Alter, Wachen, Schlafen, Gesundheit oder Krankheit. Man denke an so differente Erscheinungen ein und desselben Lebewesens wie den Samen einer Rose und ihre Blüte. Als erklärendes Prinzip zielt die Psyche als »erste Entelechie « auf das im-Ziel-Sein: auf den adulten gesunden und aktiven Reifezustand eines Lebewesens.

Mit der Formel in De anima II 1 (DA 412a19-21) ist die Psyche wie gesagt nur ihrem »allgemeinsten Begriff« nach bestimmt. ${ }^{10}$ Um den Term $\psi v \chi \eta \dot{~ f u ̈ r ~ k o n k r e t e ~ E r k l a ̈ r u n g e n ~}$ anwenden zu können, unternimmt Aristoteles einen »neuen Anlauf der Untersuchung «:

Wir sagen nun, indem wir einen neuen Anfang der Untersuchung nehmen, daß das Beseelte gegenüber dem Unbeseelten durch das Leben bestimmt ist. Da aber das Leben ( $\zeta \tilde{\eta} v)$ [eines Lebewesens] in mehrfacher Bedeutung verstanden wird, sagen wir, [das Lebewesen] lebe, wenn Leben auch nur in einer [sc. einzigen dieser] seiner Bedeutungen vorliegt: als Geist,

9 Vgl. Charles (1997: 63-84); Cho (2003); Cho (2010: 299-314); Meyer (2016a: 59-86).

${ }^{10} \mathrm{Vgl}$. Matthews (1992: 185-194, 185): »Aristotle seems to have been the first thinker to try to understand what it is to be a living thing by reference to a list of characteristic ,life functions' (or, as he called them, ,psychic powers' or ,soul-powers' - dunameis tēs psuchēs) «. 
als Wahrnehmung, als Bewegung und Ruhe dem Orte nach, ferner als Bewegung gemäß der Ernährung, als Vergehen und als Wachstum. (Arist. de An. II 2, 413a20-27).

Gemäß dieser Formel muß ein natürlicher Körper mindestens eines der folgenden Merkmale (»Lebensfunktionen«) aufweisen, wenn von »Leben« die Rede ist:

- Geist, Intelligenz (Nous: voũc),

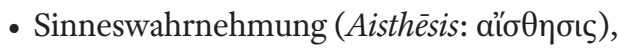

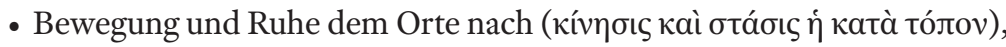

- Bewegung gemäß der Ernährung,

- Vergehen (Phthisis: $\varphi \theta$ Өíoıs),

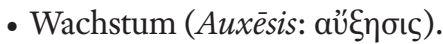

Die Liste der Lebensfunktionen ( $\delta \cup v a ́ \mu \varepsilon ı \varsigma ~ \tau \tilde{\eta} \varsigma ~ \psi v \chi \tilde{\eta} \varsigma$ ) hat einen doppelten Sinn. Erstens unterscheidet Aristoteles hier Lebewesen von unbelebten Körpern. Die Liste bietet »Minimalbedingungen « (Polansky 2007: 151), um zu testen, ob etwas lebt oder nicht.

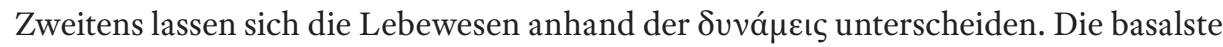

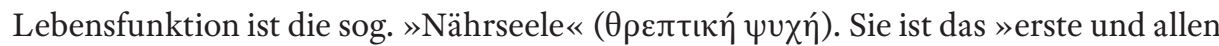
[Lebewesen] gemeinste Vermögen« (de An. II 4, 415a22-b2). Die Nährseele umfaßt drei Funktionen: Wachstum, Ernährung und Reproduktion. Bei den Pflanzen liegen diese Funktionen untrennbar zusammen vor. Das Reproduktionsvermögen ist die »natürlichste « aller Lebensfunktionen:

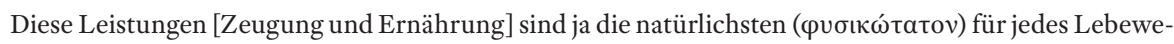
sen, insofern es vollendet ist (und nicht verstümmelt oder spontan erzeugt wird), nämlich ein anderes,

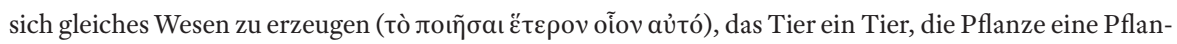
ze, damit sie am Ewigen und Göttlichen nach Kräften teilhaben; denn alles strebt nach jenem, und um

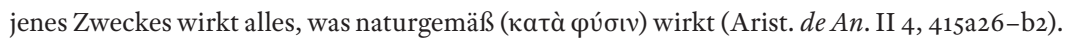

Das Reproduktionsvermögen kommt ausnahmelos allen Lebewesen zu. Reproduktion ist das Wesensmerkmal von Leben überhaupt. Tiere unterscheiden sich von Pflanzen

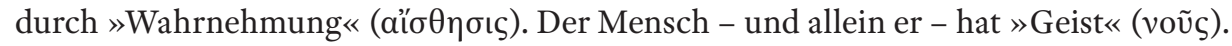
Der aristotelischen Biologie zielt darauf, die einzelnen Lebensfunktionen artspezifisch zu erklären. Die Funktionen determinieren die Physis eines Individuums. Sie bestimmten ein Individuum zu dem, was es artspezifisch ist - dazu, daß es artspezifisch so ist, wie es ist. Die Aisthesis ist wiederum Voraussetzung für weitere Dynameis wie Phantasie, Gedächtnis, Wachen, Schlafen, das Strebevermögen (Begehren, Thymos, Wille) und mittelbar auch für die animalische Fortbewegung (Frede 1992: 93-107).

Aristoteles begreift die Psyche nicht nur als ein erklärendes Prinzip, sondern auch als Ursache (Meyer 2012: 115-142). Da die Psyche die Artform (das عĩסo૬) eines jeden Wesens bestimmt, ist sie ursächlich dafür, daß ein Lebewesen so ist, wie es ist. Der spezifische Bauplan (die Artform), die sich von einem Individuum X der Spezies Y auf die artgleichen 
Individuen $\mathrm{x}_{1}, \mathrm{x}_{2}, \mathrm{x}_{3}$ usw. überträgt, ist (a) ursächlich dafür, daß diese Individuen dieselbe (formale) artspezifische Natur haben. Die artspezifische Form gibt (b) das »Ziel« (telos) jeder ontogenetischen Entwicklung vor. Lebewesen sind daher stets »teleonomisch « organisiert. So sagt Aristoteles, die Psyche sei sowohl causa formalis als auch causa finalis der Lebewesen (Seidl 1968). Hier zeigt sich, daß das Prinzip der Artenkonstanz (»Ein Löwe zeugt stets einen Löwen«) eine Grundannahme der aristotelischen Biologie ist. (Evolutionstheoretische Thesen mit Aristoteles zu vermischen, ist abwegig). Die einzelnen Spezies laufen gleichsam wie konzentrische Kreise nebeneinander her. Es gibt bei Aristoteles keinen übergreifenden Transfer von einer Spezies zu einer anderen. Akzeptiert man den Vorschlag, die genannten Passagen aus De anima als eine der eigentlichen Explanation vorgelagerte Prinzipienforschung zu lesen, so ergibt sich folgende (freilich nur schematische) Systematik der biologischen Schriften des Aristoteles:

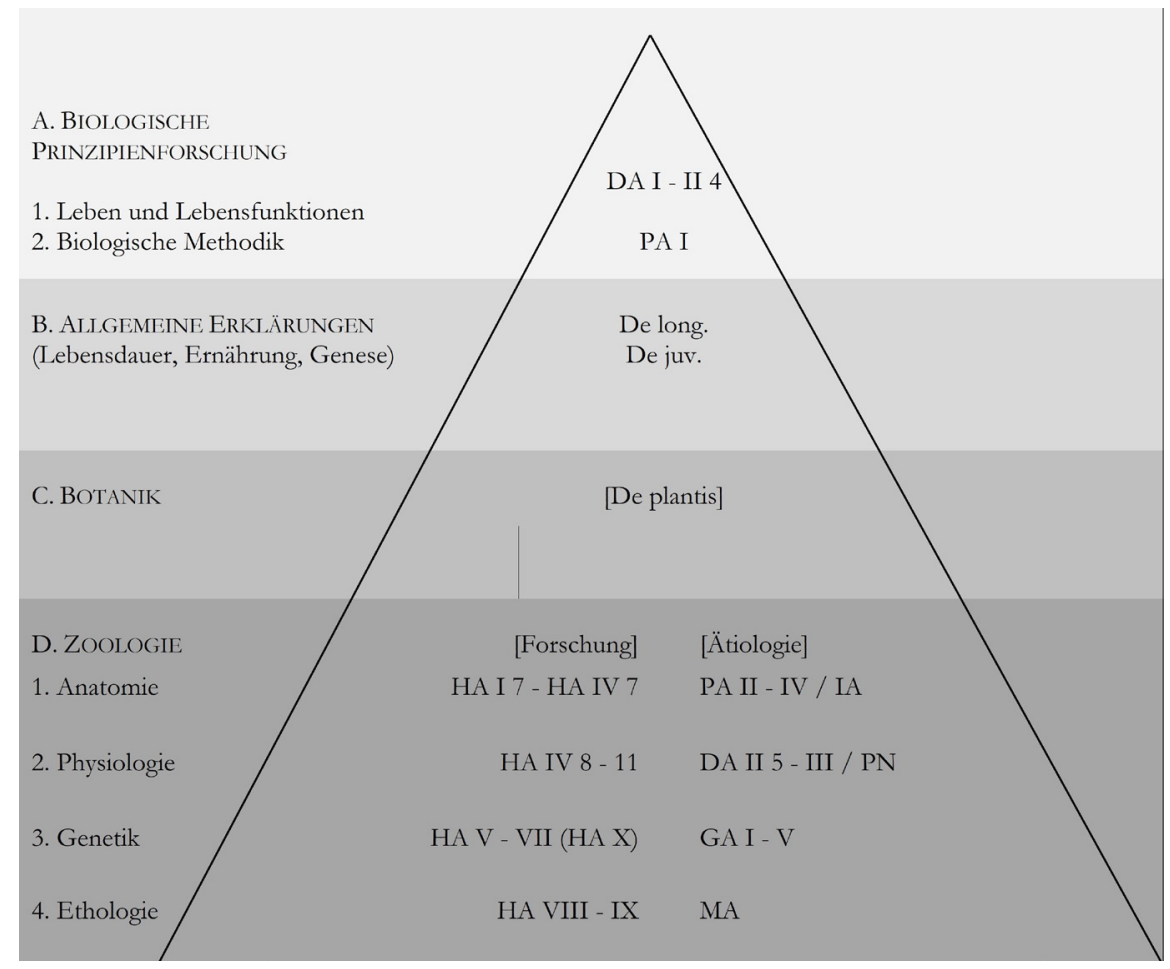




\section{Botanische Erklärungen}

An gut 430 Stellen im Corpus Aristotelicum finden sich Äußerungen zu Pflanzen. ${ }^{11}$ Insgesamt erwähnt Aristoteles ca. 6o Pflanzenspezies. Die mit Abstand meisten Arten begegnen in der Historia animalium. ${ }^{12}$ Die folgende Tabelle gibt einen Überblick über die etwa 60 von Aristoteles außerhalb von De plantis erwähnten Pflanzenarten. Der Katalog wurde auf der Grundlage des von Aubert und Wimmer (1992a: 184-187) in der Einleitung zur Historia animalium angeführten Pflanzenverzeichnisses erstellt, aber wesentlich modifiziert und erweitert. ${ }^{13}$

\begin{tabular}{|c|c|c|}
\hline 1. & ä́ros & Mönchspfeffer - Vitex agnus L. \\
\hline 2. & 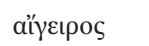 & Schwarzpappel - Populus nigra L. \\
\hline 3. & 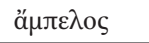 & Wein - Vitis vinifera $L$. \\
\hline 4. & á $\mu v ́ \gamma \delta \alpha \lambda a$ & Mandelbaum - Amygdalus communis L. \\
\hline 5 . & äлা०ৎ & Birnbaum - Pirus communis L. \\
\hline 6. & äpov & Aronstab - Arum dioscoridis Sibth. L. \\
\hline 7. & ả $\sigma \varphi o ́ \delta \varepsilon \lambda \circ \varsigma$ & Asphodel - Asphodelus ramosus L.* \\
\hline 8. & 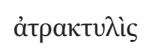 & Färberdistel - Carthamus lanatus $L .{ }^{*}$ \\
\hline 9. & ảxpás & Weidenblättrige (Holz-) Birne - Pirus salicifolia L. \\
\hline 10. & Bpúov & Je nach Kontext: Moos, meist Tang \\
\hline
\end{tabular}

${ }^{11}$ Vgl. Zeller (1862: 394-398): Zeller bietet eine gründliche Synopsis zu allen relevanten Stellen.

12 Vgl. Aubert, Wimmer (1992a: 184-187). Pflanzen sind in der Historia animalium nicht eigentlich thematisch. Entsprechende Notizen fallen in den Kontext zoologischer Observationen. Pflanzen werden in der Historia animalium v.a. erwähnt, um die Habitate oder die Nahrung der Tiere zu bestimmen. Gelegentlich werden Tiere mit Pflanzen verglichen (so die Eier des Oktopus mit Trauben). Viele Aussagen gehen auf eigene Anschauung zurück. Daß etwa Bienen bei jedem Ausflug nur eine einzige Spezies (Viola odorata) angehen, wird Aristoteles sich nicht von fremdem Munde berichtet haben lassen. Überhaupt scheint er Honig gemocht und viele Sorten verglichen zu haben.

13 Vgl. Meyer (2015a - Tabelle 10) mit sämtlichen Stellenangaben in Aristoteles' Schriften und entsprechenden Ausführungen. Bernhard Herzhoff hat mit seinem fachkundigen Rat geholfen, mannigfache Irrtümer zu vermeiden und die nachstehende Liste auf den modernen Forschungsstand zu bringen. Ihm gilt mein ausdrücklicher Dank. 


\begin{tabular}{|c|c|c|}
\hline 11. & 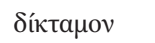 & Kretischer Origano - Origanum Dictamnus $L .{ }^{*}$ \\
\hline 12. & 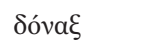 & Rohrglanzgras - Phalaris arundinacea / Donax arundinacea \\
\hline 13. & $\delta \rho \tilde{\varsigma}$ & Eiche (Stein- oder Korkeiche) - Quercus ilex o. Quercus suber \\
\hline 14. & 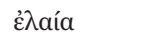 & Olive - Olea europea $L$. \\
\hline 15 . & غ̇лі́лєтроv & Hauswurz - Sempervivum marmoreum bzw. Sempervivum reginae-amalia \\
\hline 16. & 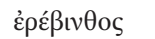 & Kichererbse - Cicer arietinum L.* \\
\hline 17. & 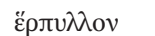 & Quendel - Thymus serpyllum L.* \\
\hline 18. & $\theta u ́ \mu o v$ & Thymian - Satureja capitata L.* \\
\hline 19. & iov & Duftveilchen - Viola odorata* \\
\hline 20. & ił́ós & Weißbeerige Mistel - Viscum album $L$. \\
\hline 21. & iтća & Weide - Salix alba / Salix purpurea \\
\hline 22. & 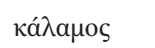 & Schilfrohr - Phragmites australis und/oder Arundo Donax \\
\hline 23. & 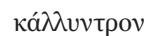 & Nicht identifizierbare Blütenpflanze \\
\hline 24. & каv入íov & Nicht identifizierbare Wasserpflanze \\
\hline 25 . & ке́үХроৎ & Hirse - Panicum miliaceum $L .{ }^{*}$ \\
\hline 26. & 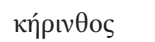 & Nicht identifizierbar \\
\hline 27. & $\kappa ı v v a ́ \mu \omega \mu о \nu$ & Zimt - Cinnamomum cassia \\
\hline 28. & KIтTós & Efeu - Hedera helix* \\
\hline 29. & $\kappa \nu \tilde{\eta} \kappa о \varsigma$ & Saflor/Färberdistel - Carthamus tintorius L.* \\
\hline 30. & кóvขל̧a & Spezies der Familie der Korbblütler - Dittrichia viscosa [Inula viscosa] \\
\hline 31. & кра́ $\mu \beta \eta$ & Kohl-Brassica oleracea L.* \\
\hline
\end{tabular}




\begin{tabular}{|c|c|c|}
\hline 32. & $\kappa \rho ı \eta ́ ~$ & Gerste - Hordeum vulgare \\
\hline 33. & 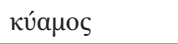 & Ackerbohne - Vicia faba L.* \\
\hline 34 . & $\kappa u v a \kappa a ́ v \theta \eta$ & Nicht identifizierbar \\
\hline 35 . & 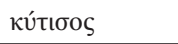 & Schneckenklee - Medicago arborea L.* \\
\hline 36. & $\lambda \varepsilon \dot{\kappa} \eta$ & Silberpappel - Populus alba \\
\hline 37. & $\mu \varepsilon \lambda \hat{\lambda} \lambda \omega \tau$ то & Honigklee - Melilotus officinalis / M. cretica \\
\hline 38. & $\mu \eta ́ \kappa \omega \nu$ & Schlafmohn o. Klatschrose - Papaver somniferum L. o. Papaver Rhoeas L.* \\
\hline 39. & $\mu v \rho \rho i ́ v \eta$ & Gemeine Myrte - Myrtus communis \\
\hline 40 . & oiváv $\theta \eta$ & Kleine (o. knollige) Mädesüß - Filipendula vulgaris* \\
\hline 41. & ópíyavov & Scharfer Dosten - Origanum heracleoticum L.* \\
\hline 42. & ópoßos & Linse - Vicia Ervilia* \\
\hline 43. & $\pi a \rho \delta a \lambda ı \alpha \gamma \chi \varepsilon ́ \varsigma$ & Giftgewächs? \\
\hline 44 . & $\pi \varepsilon u ́ \kappa \eta$ & Pinie - Pinus nigra \\
\hline 45. & $\pi \eta ́ \gamma a v o v$ & Weinraute - Ruta graveolens / Ruta montana* \\
\hline 46. & лıкрі́с. & Bitterkraut - Helminthia echioides \\
\hline 47. & лóa & allgemein für Krautpflanzen (kann auch Gräser umfassen) - Poales \\
\hline 48. & 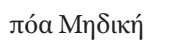 & Medisches Gras - Medicago sativa \\
\hline 49. & 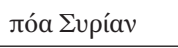 & Syrisches Gras \\
\hline 50. & $\pi \tau \varepsilon \lambda \varepsilon ́ \alpha$ & Feldulme - Ulmus minor / Ulmus campestris \\
\hline 51. & jópavos & Gartenrettich - Raphanus sativa L.* \\
\hline 52. & бíkvov & Gurke - Cucumis sativus \\
\hline
\end{tabular}




\begin{tabular}{|c|c|c|}
\hline 53. & $\sigma i ́ \lambda \varphi$ & Siliphium [heute verschwunden] \\
\hline 54. & 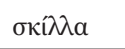 & Meerzwiebel/Blaustern - Scilla maritima $\mathrm{K} .{ }^{*}$ \\
\hline 55. & 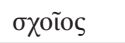 & Binsen-Juncaceae \\
\hline 56. & бта́ртоv & Binsenpfriemkraut - Spartium junceum L.* \\
\hline 57. & 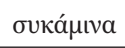 & Schwarze Maulbeere - Morus nigra \\
\hline 58. & $\sigma \cup \kappa \tilde{\eta}$ & Feige - Ficus carica \\
\hline 59. & $\varphi \lambda \varepsilon \omega ́ \varsigma$ & Besengintser - Sarothamnus scoparius \\
\hline 60. & 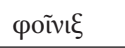 & Dattelpalme - Phoenix dactylifera \\
\hline 61. & фи̃коร & Div. Spezies von Tang (siehe auch ßpúov) / Seegras - Posidonia oceanica \\
\hline 62. & $\tilde{\omega} \times p \circ \varsigma_{5}$ & Erbse - Pisum ochrus \\
\hline
\end{tabular}

Für Aristoteles sind Pflanzen Lebewesen. ${ }^{14}$ Dies war zu seiner Zeit nicht selbstverständlich. Für einige Denker waren die $\varphi$ tá »erdverwurzelte Tiere «. Andere glaubten, etwas lebe nur, wenn es atme. ${ }^{15}$ Aristoteles betont, in der Zoologie und der Botanik kämen prinzipiell dieselben Methoden zur Anwendung. ${ }^{16}$ Er begründet dies damit, daß Tiere und Pflanzen prinzipiell aus denselben Bestandteilen bestehen. Für diese Einheit der Methode spricht zudem, daß Aristoteles' Freund Theophrast in seinen umfangreichen botanischen Schriften ähnlich vorgeht wie Aristoteles. Es ist in der Forschung unstrittig, daß die Freunde in der Biologie »arbeitsteilig « vorgingen. Aristoteles war primär Zoologe, Theophrast primär Botaniker. Aristoteles spricht ausdrücklich von den »Organen « einer Pflanze. So bezeichnet er die Wurzeln einer Pflanze als ihren Mund. Sein imposantes zoologisches Werk sollte nicht vergessen lassen, daß er eine botanische Schrift verfaßt hat, auf die er an vielen Stellen seines Werkes verweist. In dieser (durch mehrfache Übersetzung und Rückübersetzungen verformten) Schrift De plantis ${ }^{17}$ untersucht

14 Vgl. (paradigmatisch) Arist. Juv. 467b18-25. »Insofern aber etwas lebt, muß es nicht unbedingt ein Tier

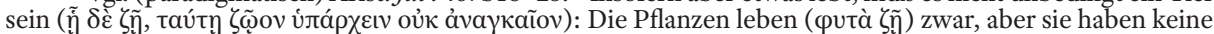
Sinneswahrnehmung. Und gerade durch die Wahrnehmung unterscheiden wir das Tier vom nicht-tierischen

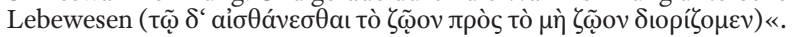

15 Vgl. Herzhoff (1999: 13-49); Meyer (2013: 107-145).

${ }^{16}$ Vgl. Wöhrle (1985); Wöhrle (1997: 387-396).

${ }_{17}$ Vgl. Drossaart Lulofs, Poortman (1989); Herzhoff (2006: 69-108); Repici (2009: 77-94). 
er pflanzliche Lebensfunktionen wie Wachstum, Ernährung, Fortpflanzung im Kontext externer Faktoren wie Bodenbeschaffenheit, Düngemittel, Wasserqualität oder Klima. Aristoteles beobachtet die auffällige Langlebigkeit vieler Pflanzen, insb. der Bäume. Die Dattelpalme (Phoenix dactylifera) gilt ihm als eines der langlebigsten Lebewesen. Zu den bahnbrechenden Erkenntnissen der aristotelischen Botanik gehört die Unterscheidung von männlichen und weiblichen Pflanzen. Ähnlich wie Charles Darwin hat sich Aristoteles mit wachsender wissenschaftlicher Erfahrung stetig mehr für botanische Fragen interessiert. Eine vorzügliche Zusammenschau wichtiger botanischer Theoreme liefert der Aufsatz von Georg Wöhrle Aristoteles als Botaniker (1997).

\section{Zoologische Erklärungen}

Das primäre Interesse der aristotelischen Biologie galt den Tieren. Mehr als 95 Prozent der Texte betreffen zoologische Fragen. In den zoologischen Schriften wird die von W. Kullmann schon 1974 konstatierte Zweiteiligkeit (Faktenerklärung - Aitiologie) fast überall mustergültig durchgeführt. Ausgangspunkt und Basis der Zoologie sind die Bücher der Historia animalium. Das Werk ist mit 152 Bekker-Seiten und zehn Büchern das umfangreichste Werk im Corpus Aristotelicum. (Das gilt selbst dann, wenn das sicher unechte Buch X und das eventuell unechte Buch VII entfallen). Heute ist unstrittig, daß Aristoteles die Historia animalium in der Zeit seines Exils nach Platons Tod ausgearbeitet hat. Allein in der Historia animalium erwähnt er ca. 550 Tierarten (Aubert, Wimmer 1992). Gegliedert nach Tierklassen ergibt sich folgendes Bild:

- Säugetiere: 75 Spezies

- Vögel: 192 Spezies

- Amphibien und Reptilien: 22 Spezies

- Fische: 134 Spezies

- Mollusken [Cephalopoden] ${ }^{18}: 10$ Spezies

- Crustacea: 18 Spezies

- Insekten: 83 Spezies

- Schaltiere (bzw. ähnliche Tiere): 39 Spezies

Es geht Aristoteles nicht um zoologische Systeme. (Das Ziel einer Systematisierung der Tier- und Pflanzenwelt entstand erst in der Neuzeit - es war gleichsam die Antwort auf die Frage nach der mit der »Entdeckung der neuen Welt« einhergehenden, bald unübersehbaren Vielfalt unzähliger Tier- und Pflanzenspezies; Mayr 2002: 82-98). Aristoteles spricht aber gelegentlich von übergreifenden Klassen - von »großen Gattungen« ( $\gamma \varepsilon ́ v \eta$

18 Vgl. Aubert (1862); Scharfenberg (2001). 


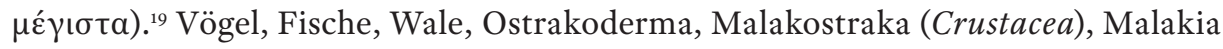
(Mollusca) und das Genos der Insekten bilden gemäß Historia animalium I 6. 49ob715 solche »großen Gattungen«. Eine generelle Einteilung der »übrigen Tiere « sei aber nicht sinnvoll (HA 49ob15-21). Die folgende Tabelle bietet eine Übersicht. Die »großen Gattungen « sind nachstehend mit Sternchen* gekennzeichnet. In Klammern steht die Zahl der Spezies gemäß Aubert und Wimmer.

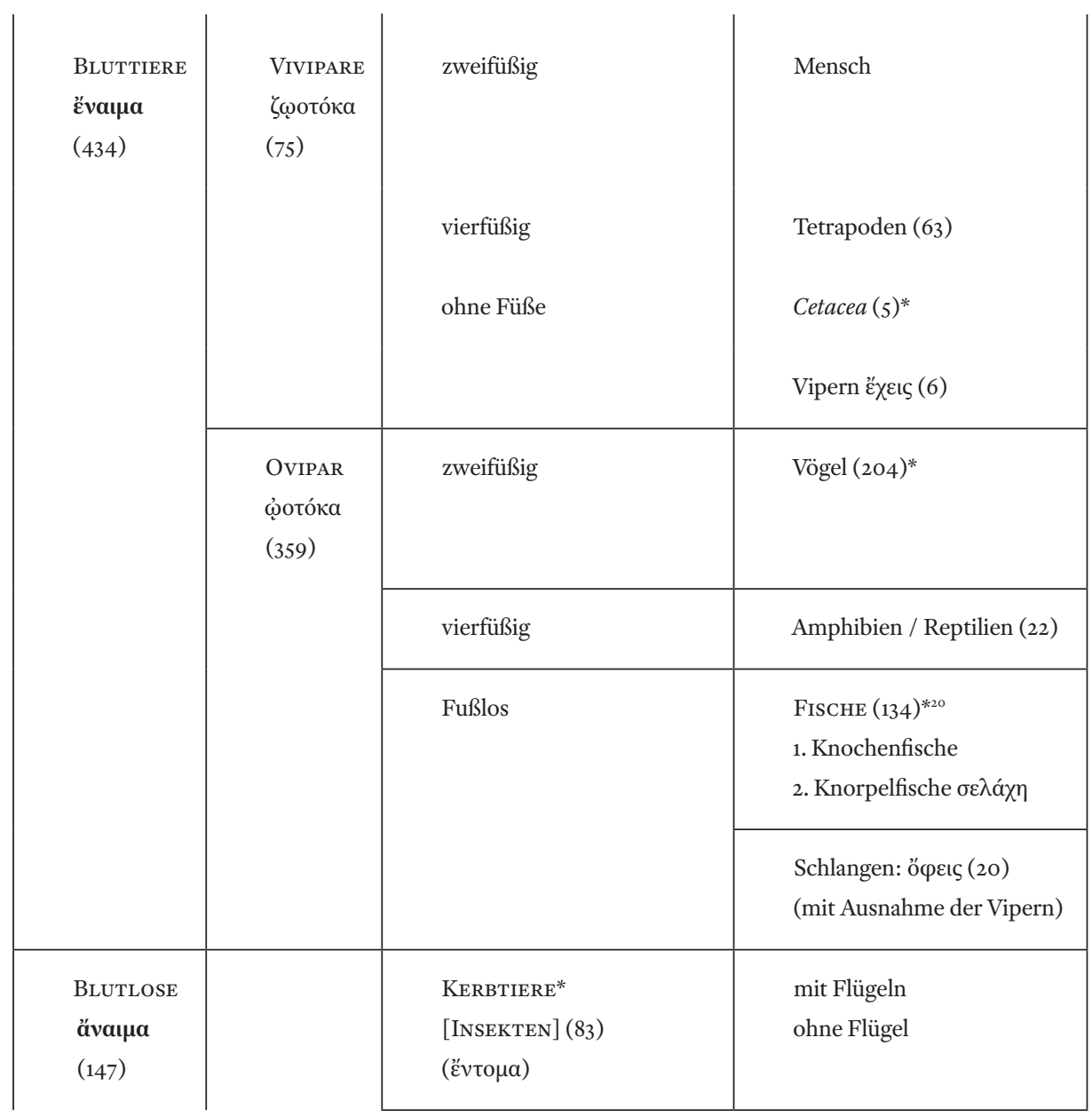

19 Vgl. Kullmann (2007: 196-210); Zierlein (2013: 68-69) zu Aristoteles' Einteilung der Tierarten.

${ }^{20}$ Vgl. Kullmann (2007: 209 f.) zur Differenzierung dieser beiden Fischgruppen. 


\begin{tabular}{|c|c|}
\hline $\begin{array}{l}\text { WEICHTIERE }^{21} \\
\text { [MOLLUSCA] (7) } \\
\text { ( } \mu \alpha \lambda \text { á́ıı })\end{array}$ & 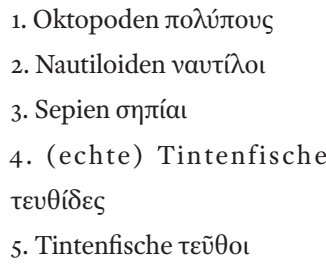 \\
\hline 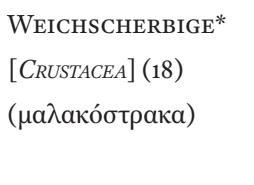 & 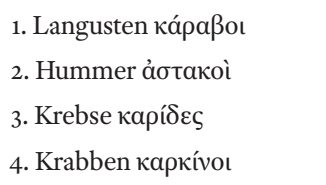 \\
\hline 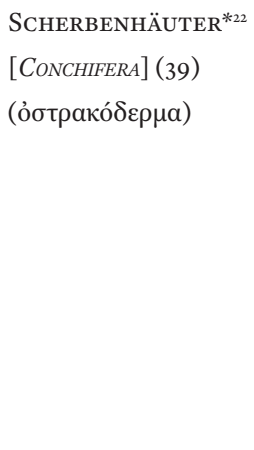 & 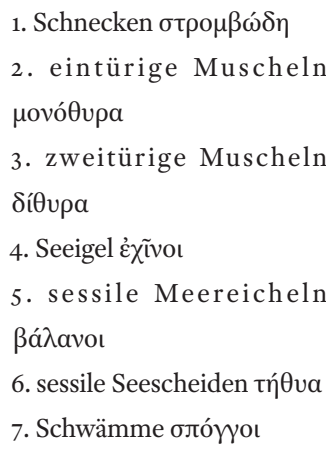 \\
\hline
\end{tabular}

Grundlegend und ubiquitär durchgehalten ist die Einteilung in blutführende ("̌v vaı $\mu$ ) und blutlose (ävaı zu Beginn des 19. Jahrhunderts eingeführten) Gliederung in Vertebraten und Invertebraten (Hirschberger 2001: 61-71). Aristoteles' Biologie geht es nirgends um eine letztgültige Systematik der Tiere. Der Gedanke einer Taxonomie ist ihm fremd. Stets stehen explanatorische Absichten im Mittelpunkt. Die Historia animalium gliedert sich (im Anschluß an

${ }^{21}$ Vgl. Aubert (1862); Scharfenberg (2001: 20-32); Scharfenberg 2015 (Anmerk. zu Kap. 2.5.4).

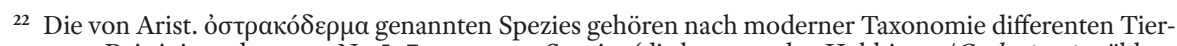
stämmen an. Bei einigen der unter Nr. 5-7 genannten Spezies (die heute zu den Hohltieren/Coelenterata zählen, ist Arist. PA IV 5, 681 a9 ff. unsicher, ob es sich überhaupt um Tiere handelt. 
eine allgemeine Einführung in die Zoologie: $H A$ I 1-6) in vier Hauptabschnitte:
A. Vergleichende Anatomie (HA I 7-IV 7)
B. Physiologische Unterschiede der Tiere (HA IV 8-11)
C. Reproduktion der Tiere (HA V-VII)
D. Verhalten, Charaktere und Krankheiten der Tiere (HA VIII und IX)

Dies sind die vier Kerndisziplinen der aristotelischen Zoologie. Auch in dieser Hinsicht ist die Historia animalium das Fundament der zoologischen Erklärung. Die ätiologischen Passagen greifen dann wie Zahnräder in entsprechende Abschnitte der Historia animalium (vgl. Schaubild oben).

\subsection{Vergleichende Anatomie}

Die vergleichende Anatomie ist mit insg. acht Büchern neben der Genetik die größte Disziplin der aristotelischen Biologie. Thematisch ist die vergleichende Anatomie in den Büchern Historia animalium I-IV und den Büchern De partibus animalium II-V. In der Historia animalium klärt Aristoteles die anatomischen Fakten (das »Dass «), in De partibus animalium gibt er funktionale Ursachenerklärungen; es geht hier um das anatomische »Warum«. (Warum hat ein Fisch Flossen?) In der Historia animalium steht die vergleichende Anatomie mit guten Gründen am Anfang. Sie eignet sich als Methode zur Erforschung aller Tiere, weil »alle Tiere Teile haben« (HA I 6). Für die weiteren Analysen wegweisend ist die auf Anaxagoras zurückgehende Unterscheidung von gleichartigen und ungleichartigen Teilen.

- Gleichartige Teile (Homoiomere) sind Teile wie Blut, Fett, Talg oder Haut, die nach antikem Verständnis, wenn man sie teilt, wieder dieselbe Substanz zeigen. In der Aristotelischen Biologie sind die Homoiomeren etwa gleichbedeutend mit »Geweben «.

- Ungleichartige Teile (Anhomoiomere) wie Augen, ${ }^{23}$ Nase, Ohren, Beine, Arme sind Teile, die sich nicht teilen lassen, ohne daß sie ihre anatomischen Funktionen verlieren. In der aristotelischen Biologie sind die Anhomoiomeren in etwa gleichbedeutend mit den $»$ Organen $\ll .{ }^{24}$

Die Historia animalium behandelt erst (a) die äußeren Organe des Menschen. Leitend ist, wie in all seinen Wissenschaften, die Devise, mit den bekannten Dingen zu begin-

${ }^{23}$ Vgl. Oser-Grote (1997: 333-349) zu Auge und Sehen nach Aristoteles und der hippokratischen Schrift De carnibus.

${ }^{24}$ Vgl. Kullmann (2007); Meyer (2016: 37-61). 
nen. Im Fall der »äußeren Organe « ist dies der Mensch (Meyer 2006: 19-34). Aristoteles geht dann (b) über zu den äußeren und inneren Organen der blutführenden Tiere, um (c) in Historia animalium III die Homoiomeren und (d) in Historia animalium IV 1-7 die Teile und die vier Hauptgruppen der blutlosen Tiere zu behandeln. Die aitiologischen Passagen in De partibus animalium II-IV gliedern sich nach Art, Qualität und Funktion der Teile bzw. nach topologischen Aspekten (Balme 1972). Nach der Einführung (PA II 1-3) zur Zusammensetzung (»Synthesis«) der Tiere und dem Verhältnis ihrer Teile zu den Grundqualitäten >warm<, >kalt<, >feucht< und >trocken $<$ handelt De partibus animal ium II 1-9 zunächst allgemein von den Homoiomeren. De partibus animalium II 10-III 12 untersucht dann die Anhomoiomeren. Der Gedankengang folgt in diesem Kontext (ähnlich wie Historia animalium I) topologischen Gesichtspunkten. Zunächst werden (nach dem wichtigsten Organ: dem Herzen) die am Kopf liegenden Organe besprochen. Es folgen die im Hals ansässigen Teile, die in der Brustgegend liegenden Organe, schließlich die Organe der Bauchgegend. In De partibus animalium IV 1-14 handelt Aristoteles von den Besonderheiten der Anhomoiomeren. Der Weg in De partibus animalium führt mithin vom Allgemeinen zum Besonderen. Am Ende behandelt Aristoteles Organe, die (wie das Spritzrohr der Cetacea) bei nur wenigen Spezies vorkommen (Kullmann 2007). Als Ergänzung zu Departibus animalium ist (obgleich wohl früher verfaßt) die acht Seiten kurze Schrift De incessu animalium zu lesen (Kollesch 1985). Wie im Eingangssatz betont, geht es hier allein um die zur animalischen Lokomotion nötigen Organe. Kerngedanke: Für jede tierische Ortsbewegung muß ein spezifisches Moment der Ruhe (innerhalb wie außerhalb des Körpers) gegeben sein. Hier kommen also physikalische bzw. mechanische externe Faktoren (z.B. Ziehen und Stoßen) zur Sprache. In De incessu animalium zeigen sich die Grenzen der Anatomie. Die Erklärung der tierischen Fortbewegung bedurfte der zusätzlichen Erörterung physiologischer und ethologischer Faktoren. Die kleine Schrift De motu animalium behandelt interne Faktoren der animalischen Fortbewegung. Sie gehört ebenfalls nicht auf das Gebiet der Anatomie.

\subsection{Physiologie}

Auch in der Physiologie gibt die Historia animalium die Leitthemen vor. Die Pragmatie HA IV 8-11 ist relativ kurz und erscheint im Gesamtkontext wie ein Exkurs. Wieder klärt Aristoteles zunächst die Fakten. Er behandelt die Unterschiede der Tiere hinsichtlich der vorhandenen Sinnesorgane und Stimmwerkzeuge - und fragt, welche Tiere wachen, schlafen und träumen. Die Parva naturalia liefern dann zu diesen Themen dann entsprechende kausale Erklärungen (King 2011: 97-105). Historia animalium IV 8 untersucht das Vorhandensein der fünf Sinne. Demnach haben fast alle Tiere sämtliche Sinne. Historia animalium IV 9 erörtert das Vorhandensein von Ton (భó $\varphi \circ \varsigma$ ), Stimme ( $\varphi \omega v \eta ́)$ und Sprache ( $\delta$ เá $\lambda \varepsilon \kappa \tau o \varsigma)$ (vgl. Meyer 2011). Zwar ist jeder Stimmlaut ein Ton. Nicht jeder Ton aber ist ein Stimmlaut. Jede gesprochene Äußerung bedarf der Stimme. Nicht jede Stimme aber ist Sprache. Das Sprechen bedarf nicht nur der Stimme, sondern weiterer Organe 
zur Artikulation bedeutungshafter Laute. Es fordert zudem eine intentionale Steuerung dieser Organe. Historia animalium IV 10 behandelt Wachen, Schlafen und Träumen; Kap. IV 11 die Geschlechterdifferenzen. Gemäß Historia animalium IV 10 schlafen und träumen alle vierfüßigen Tiere. Das Thema der Sinnesphysiologie nimmt De anima II 5-III 13 (unter Einschluß eines kurzen Exkurses über den Geist) in einem prinzipiellen Sinne wieder auf. Es wird in der kleinen Schrift De sensu et sensibilibus unter zoologischen Aspekten fortgeführt. Daß Aristoteles der Sinnesphysiologie viel Aufmerksamkeit widmet, liegt daran, daß die Aisthesis die Grenze zwischen Tieren und Pflanzen markiert und eine Voraussetzung ist für Funktionen wie Phantasie, Gedächtnis, Streben und Ortsbewegung. Damit verbindet sich die Frage, welche internen Ursachen für die animalische Lokomotion verantwortlich sind (Corcilius 2008). Die übrigen physiologischen Abhandlungen reihen sich wie eine Perlenkette kleiner Meisterwerke aneinander. Nach De sensu et sensibilibus folgt De memoria et reminiscentia (Cessi 1997). Aristoteles entwickelt in 453a1of. die These, das bewußte Erinnern sei »gleichsam eine Art Schlußfolgerung « (King 2004: 142 f.). Wie in De anima II 5-III 13 ist die Grenze zwischen Tier und Mensch thematisch. Dieses Problem tangiert er ebenfalls in den Schriften Über Wachen und Schlafen und Über die Träume. Die Schrift De divinatione per somnum ist eine Art Exkurs zu einem noch bei Cicero kontrovers diskutierten Thema (vgl. van der Eijk 1994: 35-93). Die folgenden Schriften der Parva naturalia (De longaevitate, De juventute et senectute und der zu dieser Schrift zugehörige Traktat De vita et morte) gehören nicht eigentlich auf das Gebiet der Physiologie. Sie fallen, da Aristoteles hier Tiere und Pflanzen gleichermaßen behandelt, aus dem engeren Rahmen der Zoologie und widmen sich Fragen der allgemeinen Lebenserklärung (King 2004: 97). De respiratione hat mit zehn Bekker-Seiten die Länge eines durchschnittlichen aristotelischen Buchs. Sie ist als geschlossene Abhandlung konzipiert (Meyer 2013: 31-59) und beginnt mit einer gesonderten Doxographie. Aristoteles kritisiert seine Vorgänger Demokrit, Anaxagoras, Diogenes v. Apollonia, Platon und Empedokles. Als Grund ihrer fehlerhaften Ansichten führt er an, »diese Naturforscher « hätten »keine Ahnung von Anatomie «. Sie begriffen daher »nicht, daß die Natur alles um eines Zweckes wegen macht. Hätten sie nach dem Zweck der Atmung bei den Tieren geforscht und dies an den entsprechenden Organen, Kiemen und Lungen, studiert, hätten sie die Ursache eher gefunden« (Resp. 3, 471b23-29). Wieder zeigt sich, daß Aristoteles die Basis der Erklärung in der vergleichenden Anatomie sieht. Die Kernthesen der Schrift De respiratione lauten: (a) Atmung ist kein Kriterium für animalisches oder pflanzliches Lebens. (b) Kiemenatmung ist nicht eigentlich Atmung. (c) Atmung dient wesentlich dem Zweck der Kühlung jener Tiere, die Lungen haben. Es ist daher (d) wichtig, die Atmung gleichermaßen als Ein- und Ausatmung zu begreifen (Meyer 2013). Mit De respiratione ist der Reigen der physiologischen Schriften geschlossen. 


\subsection{Genetik}

Es hatte sich schon ergeben, daß Aristoteles die Reproduktion als »natürlichste« Leistung aller Lebewesen begreift. Es erstaunt daher nicht, wenn er den Vorgängen und Bedingungen der tierischen Reproduktion und Ontogenese besondere Aufmerksamkeit widmet. In Anlehnung an die von ihm gewählte Ausdrucksweise soll dieses Gebiet hier vereinfacht > Genetik< heißen. Sie ist Thema der Bücher Historia animalium V-VII, des umstrittenen Buches Historia animalium X und der fünf Bücher der Schrift De generatione animalium. Für moderne Leser sei bemerkt, daß Erklärungen zur Vererbung bei Aristoteles einen nur kleinen (aber nicht unwichtigen) Teil dieser Thematik ausmachen (Kullmann 1998: 287-231). Auch in der Genetik ist die Historia animalium die Basis der später ausgeführten Aitiologie. Historia animalium V beginnt mit einer Auflistung differenter Fortpflanzungsmodi. Demnach gibt es:

a. sexuelle Paarung (nur bei Wesen mit Geschlechterdimorphismus),

b. Fortpflanzung ohne Geschlechterdimorphismus,

c. »spontane Entstehung« (aus Schlamm, Fäulnis etc.).

Die Unterschiede der Fortpflanzungsmodi sind anatomisch begründet. Aristoteles erforscht in De generatione animalium I erst, bei welchen Tieren welche Geschlechtsorgane vorliegen - ob also überhaupt Geschlechterdimorphismus vorliegt. In den komplementären Abschnitten Historia animalium V-VII ändert sich (gegenüber HA I-IV) die Untersuchungsrichtung. Wählte er zuvor der Weg vom Menschen zu den niedrigen Tieren, so wählt er nun den Weg vom Einfachen zum Komplexen. Er beginnt bei den Schal- und Krustentieren, geht über zu Insekten, Weichtieren und Fischen, um Reptilien, Vögel, Säugetiere und den Menschen zu behandeln. Begründung: »Von den Bipeden ist einzig der Mensch vivipar« (HA V 1, 539a14-15). Zunächst ist von der generatio spontanea als dem primitivsten Reproduktionstyp die Rede. ${ }^{25}$ Die Kapitel Historia animalium V 1-8 thematisieren die Positionen der Tiere bei der Kopulation. Historia animalium V 8-14 handelt von Paarungszeit, Geschlechtsreife und Fertilitätsdauer. Der Passus enthält reiche Informationen zur Lebensdauer vieler Arten (Meyer 2015) und Angaben zur Fischbrut, zu Paarungszeiten von Fischen, Mollusken und Vögeln. Aristoteles sagt, kein Jungtier sei von Geburt an zeugungsfähig. Mit Historia animalium V 15 beginnen die Ausführungen zu Zeugung (»Genese«) und Entwicklung. Nach den Schaltieren (und einigen Weichtieren, die er dieser Klasse nicht zuordnet) werden die Insekten (inklusive der Spinnen und Skorpione), die oviparen Tetrapoden (Schildkröten, Krokodile, Echsen) und die Schlangen behandelt. Gemäß Historia animalium V 34 sind Vipern vivipar und brüten aus. Historia animalium VI setzt die Untersuchung zur Fortpflanzung fort. Das Buch teilt sich in drei Abschnitte: Fortpflanzung (a) der Vögel; (b) der Fische inklusive

\footnotetext{
${ }^{25}$ Vgl. Capelle (1955: 150-180); Depew (2010: 285-297); Flashar (2010: 331-338).
} 
der Meeressäuger und (c) der viviparen Tetrapoden. Historia animalium VII behandelt die Fortpflanzung und Entwicklung des Menschen (Meyer 2016c).

Die Erklärung der Ursachen der in Historia animalium V-VII behandelten »Genesis « ist das eigentliche Thema von De generatione animalium. Das Werk teilt sich in fünf Bücher und faßt 75 Bekker-Seiten. Es schließt ausdrücklich an die in De partibus animalium vorgeführten anatomischen Funktionsanalysen an. Thema von De generatione animalium ist die causa efficiens in expliziter Abhebung zur causa materialis (dem Thema der Anatomie). Nach einem Proömion zur Typologie der Geschlechtsteile beginnt Aristoteles mit den Fortpflanzungsmodi, die er nach Maßgabe einer Scala naturae gemäß den Fortpflanzungsprodukten anführt. In diesem Rahmen ergibt sich folgende Stufung:

\begin{tabular}{|c|l|l|}
\hline GRADUS & QUID EST & CAUSA MATERIALIS \\
\hline 1. & $\begin{array}{l}\text { Tiere, die innerhalb ihrer selbst ihnen ähnliche Jun- } \\
\text { ge hervorbringen: alle viviparen Tetrapoden. }\end{array}$ & warm und feucht \\
\hline 2. & $\begin{array}{l}\text { Tiere, die außerhalb ihrer selbst ihnen ähnliche Jun- } \\
\text { ge hervorbringen: Knorpelfische und Vipern. }\end{array}$ & weniger warm und feucht \\
\hline 3. & $\begin{array}{l}\text { Tiere, die ein ihnen unähnliches, vollendetes Ei } \\
\text { hervorbringen: Vögel, ovipare Tetrapoden und (die } \\
\text { meisten) Schlangen. }\end{array}$ & warm und trocken \\
\hline 4. & $\begin{array}{l}\text { Tiere, die ein ihnen unähnliches, unvollendetes Ei } \\
\text { hervorbringen: } \\
\text { Fische, Ostrakoderma, Weichtiere. }\end{array}$ & weniger warm und trocken \\
\hline 5. & $\begin{array}{l}\text { Tiere, die Larven hervorbringen: Insekten (etwa } \\
\text { Heuschrecken, Spinnen, Grillen, Wespen). }\end{array}$ & kalt und trocken \\
\hline 6. & $\begin{array}{l}\text { Tiere, die »von selbst « im Schlamm oder in Fäul- } \\
\text { nis entstehen (etwa Flöhe, Mücken, Stechfliegen und } \\
\text { »ähnliche Gattungen«). }\end{array}$ & kalt und trocken \\
\hline
\end{tabular}

Er ergänzt die Überlegungen durch Beobachtungen zur Phytogenese. Bei den Pflanzen gibt es differente Reproduktionsmodi. Manche Gewächse entstehen durch Samen, andere spontan. Grundlage der genetischen Kausalerklärung ist die Anatomie. Da sich sexuelle und asexuelle Fortpflanzungsmodi unterscheiden und bei sexueller Paarung Geschlechterdimorphismus vorliegt, muß erst (im Sinne einer Erklärung des Dass) ermittelt werden, bei welchen Tieren Dimorphismus vorliegt. Geschlechterdifferenz heißt nicht, daß Männliches und Weibliches stets auf zwei Individuen verteilt ist. Bei vielen Pflanzen existieren Männliches und Weibliches in einem einzigen Individuum. Männchen und Weibchen unterscheiden sich anatomisch: »Männlich« nennt Aristoteles Tiere, die in ein anderes Wesen zeugen. »Weiblich« heißen Tiere, in die hinein gezeugt wird ( $G A$ I 2). Aristoteles analysiert, welche Geschlechtsteile es gibt und bei welchen Tieren sie vorkommen. Er unterscheidet anhomoiomere und homoiomere Teile. Anhomoiomere Geschlechtsorgane (Penis, Hoden, Samengänge, Gebärmutter) existie- 
ren meist paarig. Homoiomere Teile sind bei Männchen der »Same« (dies wird noch weiter differenziert), bei Weibchen die »Katamenien« (Monatsblutungen). De generatione animalium I 4-23 klärt die anatomischen Voraussetzungen ausführlich nach Tiergruppen (Bluttiere vs. Blutlose). Aristoteles komplettiert hier die fehlenden Abschnitte in Historia animalium und in De partibus animalium.

Daß die früheren Schriften die Geschlechtsteile nur randständig behandelt hatten, zeigt, daß er die Genetik von vornherein gesondert untersuchen wollte. Ebenfalls belegen Verweise auf entsprechende Zeichnungen, daß ihn das Thema von Beginn an beschäftigte. Bedeutsam ist seine Widerlegung der sog. Pangenesistheorie (Althoff 2006: 35-50). Gemäß dieser (im Corpus Hippocraticum entwickelten) Lehre stammt der Samen von allen Körperteilen. Aristoteles zeigt in einer musterhaften Argumentation, warum dies unmöglich ist (Althoff ebd.). Er widerspricht zudem früheren Dogmen, wonach sich Sperma auch bei Frauen bildet. Er etabliert die (zwar nicht neue, aber zu seiner Zeit kontroverse) These, das Männliche und das Weibliche hätte den gleichen Anteil an der

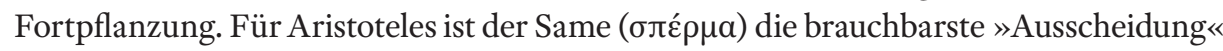

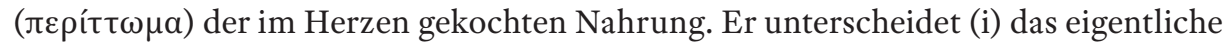
Leben tragende schaumartige Sperma und (ii) die das Sperma substantiell vermittelnde

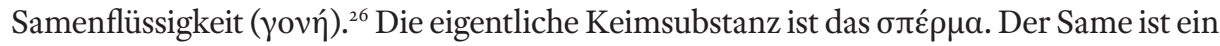
aktiv Bewegendes und insofern »wie ein Organon« $(G A 73 \mathrm{Ob})$. Die Katamenien bilden

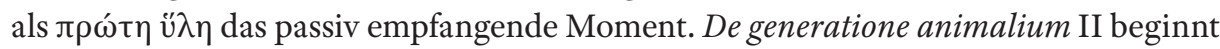
mit einer basalen Einführung zur Bedeutung der Fortpflanzung für die biologische Episteme. Aristoteles erklärt die Beschaffenheit des Samens und seine Funktion im Reproduktionsprozeß. Der Same ist Träger der Psyche (Föllinger 2005: 237-238); so der aitiologische Kern der aristotelischen Genetik. Der männliche Same verleiht wegen seiner höheren Wärme als »Bewegungsprinzip« dem weiblichen Blut wichtige Bewegungsimpulse. Aus dem Samen entwickelt sich alles in alle Körperteile hinein. Daß der Same (als materieller Träger einer den Individuen eigentümlichen »generativen Wärme«) als Träger der Psyche vorgestellt wird, ist vor dem Hintergrund zu verstehen, daß sich nicht zwei Psychen zu einer einzigen Psyche verbinden können (Althoff 1992: 181-193).

De generatione animalium II 4-III 11 behandelt die ontogenetische Entwicklung der Tiere. Aristoteles beginnt mit den Bluttieren. Ausgehend von Beobachtungen an Pflanzen und Vogeleiern untersucht er die embryonale und frühe Entwicklung des Menschen (Meyer 2016c: 25-52). Die hier vorgelegte Theorie läßt sich wie folgt skizzieren: Aristoteles vergleicht die Befruchtung mit Vorgängen der Käsegewinnung. Aus der Vereinigung

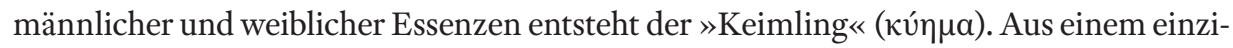
gen Keimling kann nur ein einziges Individuum entstehen. Zwillinge sind stets zweieiig ( $G A 728$ b36 f.). An sich ist der Embryo noch kein selbständig lebendes Individuum. Erst mit der Geburt kann von einem Menschen gesprochen werden: »Die Geburt ist ein Über-

\footnotetext{
${ }^{26}$ Vgl. Föllinger (2005: 237 f.); King (2005: 533 f.) zu dem bei Aristoteles gelegentlich schwankenden Wortgebrauch.
} 
gang aus dem Nichtsein in das Sein, der Tod wiederum aus dem Sein ins Nichtsein « (GA

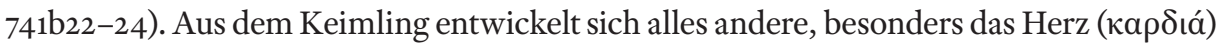
als locus animi (»Zentrum des Lebens«). Ohne das Herz wäre die Genese der anderen Teile unmöglich. Aus dem Keimling entstehen Sehnen ( $v \varepsilon \tilde{v} \rho \alpha)$ und Adern ( $\varphi \lambda \varepsilon \dot{\varepsilon} \beta \varepsilon)$ ). Sie verleihen dem Keim äußere Festigkeit. Eine der Adern ist der »sogenannte Nabelstrang « ( $G A$ 739a23). Der Nabelstrang (ỏ $\mu \varphi \alpha \lambda o ́ s)$ entsteht, sobald der Keim gebildet ist und in der Gebärmutter andockt, um den Keimling von der Gebärmutter mit Nahrung zu versorgen. Mit der Ernährung entsteht um das Herz (das Aristoteles mit einem Herd vergleicht) aus dem reinsten Ernährungsmaterial das Fleisch (GA 644a). Das Fleisch ( $\sigma a ́ \rho \xi)$ vermittelt die Tastempfindung. Im Embryo findet ein Übergang statt vom pflanzlichen zum tierischen Leben. Weitere Homoiomeren (Knochen, Haut, Haare, Nägel) bilden sich um des Fleisches wegen. Die Haut entsteht (»wie die Haut auf einer erkaltenden Suppe «) durch Abkühlung des Fleisches. Aristoteles erkannte die Funktion der Plazenta (in der modernen Biologie ein Merkmal aller echten Mammalia). Gemäß De generatione animalium II 7 sind alle Embryonen der Schwimm-, Flug- und Gangtiere von einer zarten Haut umgeben. Diese Haut trennt sie von der Gebärbutter und dem darin befindlichen Fruchtwasser. Die Knochen (ỏotéa) existieren gemäß Departibus animalium II 9 wie die Adern nicht isoliert. Sie bilden ein zusammenhängendes System - das Skelett ( $\sigma \kappa \varepsilon \lambda \varepsilon \tau o ̀ v)$. Die Knochen entstehen gemäß Kapitel II 6 aus der ersten (vom Sperma kommenden) Ausscheidung. Die Adern haben ihren Ursprung im Herzen. Die Knochen bilden sich von der Wirbelsäule her und bleiben mit ihr stets verbunden. Die Wirbelsäule garantiert die Längserstreckung der Tiere und ihre Geradheit. Ihre primäre genetische Funktion ist die Vermittlung von Wachstum. Das Knochenwachstum ist durch ein je artspezifisches Maximum limitiert. Von den Knochen bildet sich die Schädeldecke zuletzt ( $G A$ II 6). Aus demselben Material wie die Knochen bestehen die Zähne. Abgesehen von widernatürlichen Ausnahmen wird der Mensch zahnlos geboren. Für die Genese der Anhomoiomeren ist die in De generatione animalium II 5 formulierte Maxime leitend, in den frühen tierischen Organen seien die aisthetische Fähigkeiten schon notwendig angelegt. Mit der Organentstehung ist das »pflanzliche Stadium « der Embryonalgenese abgeschlossen. Beim Menschen beginnt die Organentwicklung (als zweite ontogenetische Phase) etwa 19 Tage nach der Befruchtung. In De generatione animalium II 1 notiert Aristoteles, es entstehe nicht alles gleichzeitig. Das Herz entsteht als erstes Organ: »Zuerst aber bilden sich Anfang und Mittelpunkt. Dies ist bei den blutführenden Tieren das Herz - bei den anderen Tieren etwas Analoges « (GA II 5, 741b15 f.). Gegen Demokrit argumentiert er, daß sich erst die inneren und dann die äußeren Organe bilden. In $D e$ generatione animalium II 6 (GA 642a16-18) widerspricht er der These früherer Physiologen von einer exakt bestimmbaren Abfolge der Organgenese mit dem Einwand, eine solche Abfolge könne man nicht präzise beobachten. Selbst wenn viele Dinge wegen ihrer Winzigkeit unsichtbar seien, sei klar, daß das kleine Herz noch vor der größeren Lunge

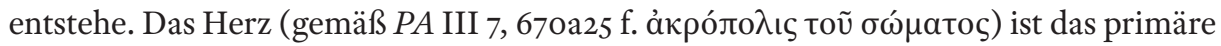
Organ, von dem her und um das herum alles andere entsteht. Die ebenfalls zur Blutkochung beitragende Leber existiert noch vor der Lunge. Für die inneren Organe ergibt 


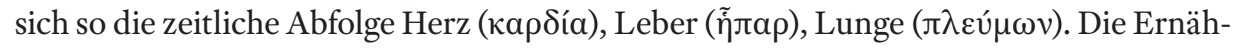
rung beginnt vor der Atmung. Die oberen Organe bilden sich früher als die unteren. Erst

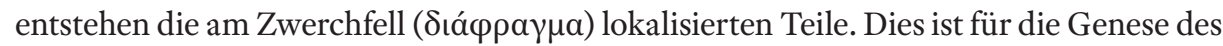
Gehirns wichtig: »Wegen der Wärme des Herzens aber bildet da, wo die [vom Herzen ausgehenden] Adern oben enden, die zu der Wärme des Herzens im Gegensatz stehende

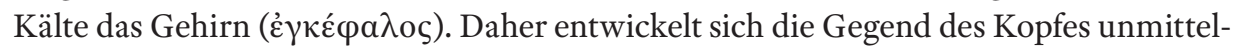
bar nach dem Herzen und übertrifft an Größe die anderen Teile, denn das Gehirn ist von Anfang an eine große flüssige Masse $(G A 743$ b27-32). Aus der Gehirnflüssigkeit bildet sich das innen flüssige, kalte Auge. Wie die Genese des Gehirns vollendet sich auch die Entwicklung erst spät. Die Augen wandern von ihrem ursprünglich dem Gehirn nahen Ort zu ihrem Ort im Gesicht. Das Auge (ó $\varphi \theta a \lambda \mu o ́ s)$ erscheint bei allen gehenden, fliegenden und schwimmenden Tieren von Anfang an sehr groß. Wie alle Säugetiere wird der Mensch mit geschlossenen Augenlidern geboren. Ihre Hauptfunktion ist der Schutz des Auges. Sie spalten sich bei der Geburt und sind erst dann beweglich. Aristoteles spielt hier auch auf Wachen und Schlafen an. Weitere Beobachtungen gelten der postnatalen Entwicklung. Erst nach der Geburt kann der Mensch sehen. Da die Natur nichts umsonst (daher nichts zur falschen Zeit) macht, sondern überall »ökonomisch« $(G A$ 744b16) agiert, wäre die Sehfähigkeit im Uterus nutzlos. Einzig beim Menschen finde ein Wechsel der Augenfarbe statt. Aristoteles entwickelt vs. Empedokles eine Theorie zur Blauäugigkeit des Säuglings. Das Ausbleiben des Farbwechsels ist Schwäche. Hinsichtlich des Embryos heißt es, der Kopf sei (wegen der großen Menge des Gehirns in Relation zum Rest des Leibes) sehr groß. Wie Platon ( $L g$. VII 789a) weist Aristoteles hin auf das in der Kindheit besonders rasche Wachstum. Gemäß De generatione animalium I 18 (GA 725b23-25) wächst der Mensch in den ersten fünf Jahren bis zur Hälfte seiner späteren Länge. Die Sprachentwicklung ist hier nicht thematisch. Die Geschlechtsreife bei Jungen beginnt mit ca. 14 Jahren; bei Mädchen, wenn sich die Brüste »etwa zwei Finger« heben. Seltsam ist, daß Aristoteles die Jünglinge erst ab dem 21. Lebensjahr für zeugungsfähig hält. Mit der Fortpflanzungsfähigkeit erreicht der Mensch sein biologisches Telos. De generatione animalium III setzt die Pragmatie zur Entwicklung der Lebewesen fort. Aristoteles beginnt mit den Vögeln. Dann behandelt er Fische und blutlose Tiere (Malakia, Malakostraka, Insekten; besonders die Bienen; vgl. Föllinger 1997). Am Schluß stehen die Ostrakoderma (bzw. ihnen ähnliche Tiere) und Ausführungen zur generatio spontanea. In den ersten Kapiteln von De generatione animalium IV untersucht Aristoteles (in kritischer Abhebung zu früheren Dogmen) die Ursachen der Geschlechterdifferenzierung. Die restlichen Kapitel tangieren das Thema der Vererbung (u.a. erblich bedingten Mißbildungen und Krankheiten). Das Buch De generatione animalium V wurde von Maria Liatsi 2002 eingehend untersucht und kommentiert. 


\subsection{Verhaltensforschung}

Das vierte Hauptgebiet der aristotelischen Zoologie ist die Verhaltensforschung (Schmitt 1997: 259-286). Auf diesem Gebiet verteilt sich die Zweiteiligkeit von Fakten- und Ursachenerklärung nicht auf verschiedene Schriften. Referenztexte sind die Bücher Historia animalium VIII \& IX. Leicht vergröbert lassen sich die Ausführungen wie folgt gliedern: Historia animalium VIII: Einfluß äußerer Faktoren auf Lebensweise und Verhalten
- Nahrung
- Wechsel der Habitate
- Winterschlaf und Winterquartiere
- Habitate und Populationsökologie
- $\quad$ Einfluß der Habitate auf das Verhalten
- $\quad$ Einfluß von Brunst und Trächtigkeit auf das Verhalten

Historia animalium IX: Einfluß innerer Faktoren auf Lebensweise und Verhalten
- Allgemeiner Charakter der Tiere
- Klugheit teils als »Nachahmung des menschlichen Lebens «
- Lernfähigkeit
- Zeichen für Klugheit

Mit Buch VIII beginnt ein neuer Abschnitt in der Historia animalium. Nachdem zuvor Anatomie, Physiologie und Reproduktion behandelt wurden, geht es nun um die »übrige Natur« der Tiere. Es spricht für die Geschlossenheit der Historia animalium, daß Aristoteles in Buch VIII auf die in Buch I genannten Unterscheidungen zurückkommt. Gemäß Historia animalium I 1 (GA 487a11-12) beruhen die Unterschiede der Tiere auf »Lebensweisen« ( $\beta$ íoı), »Verhaltensweisen «

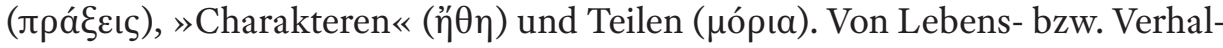
tensweisen und Charakteren spricht Aristoteles gleich im ersten Satz von Buch VIII. Gemäß Historia animalium VIII 1 (HA 588a15-18) finden sich bei den meisten Tieren Charaktermerkmale, die beim Menschen deutlicher auftreten (Meyer 2017: 141-163). Dies hinsichtlich von Zahmheit, Wildheit, Sanftmut, Heftigkeit, Kühnheit, Feigheit, Furcht, Dreistigkeit, Mut und Verschlagenheit. Bei vielen Tieren finden sich »Anzeichen « von »Gedanken« und »Verstehen « (HA 588a23). Menschen und Tiere unterscheiden sich nur graduell (»mehr oder weniger«) (vgl. Dierauer 1977). In dieser Hinsicht sind die Grenzen von Menschen und Tier also fließend. Aristoteles begründet dies so:

- Die Psyche der Kinder unterscheidet sich nicht von der Psyche der anderen Tiere.

- Die »Physis« geht generell nur allmählich vom Unbeseelten zum Beseelten über. 
- Auch die Übergänge von Pflanzen und Tieren sind fließend. Zweifel hinsichtlich solcher Grenzen herrschen besonders bei sessilen Tieren wie Steck- und Scheidenmuscheln, die abgelöst von ihrer Basis nicht überleben können.

- Ebenfalls die Ostrakoderma (Crustacea) scheinen in Relation zu den Schreittieren als unbelebt.

- Ganze Gattungen von sessilen Tieren scheinen empfindungslos zu sein. Besonders die Tethya (Wanderschwämme) und Spongien (Schwämme) gleichen den Pflanzen.

In all diesen Fällen gibt es zwischen Tieren und Pflanzen kaum merkliche Unterschiede. Aristoteles schreibt, die einzige Funktion (das Ergon) der Pflanzen liege darin, sich

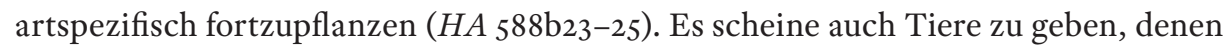
keine andere Funktion als bloß Reproduktion zukomme. Höhere Tiere zeichnen sich darin aus, daß sie sich um ihren Nachwuchs kümmern (HA 588b27-589a4). Alle Lebewesen ernähren und reproduzieren sich. Am meisten unterscheiden sich die Tiere in der Art und Weise ihrer Ernährung. Historia animalium VIII 2-11 behandelt Themen der Nahrung (Althoff 1997: 351-364). Mit Historia animalium VIII 12 beginnt ein neuer Passus. Aristoteles beschreibt »Praxis « in zoologischer Hinsicht wie folgt: »Die Praxeis derselben (Tiere) beziehen sich sämtlich auf die Begattung und auf die Erzeugung der Jungen, so wie auf die Herbeischaffung der Nahrung und auf den Schutz vor Wärme und Kälte und den Wechsel der Jahreszeiten« (HA 596b20-23). Ergänzend heißt es: »Alle Tiere haben eine angeborene Empfindung für die Veränderung der Temperatur« ( $H A$ 596b23-24). Es folgen Beobachtungen zum saisonbedingten Wechsel der Habitate der Vögel (Zugvögel sind u.a. Kraniche, Pelikane, Wachteln, Ringeltauben; nicht aber Haustauben) und Angaben zu Abflug- und Rückkehrzeitpunkten. Tiere, die ihre Orte wechseln, sind u.a. die in Historia Animalium VIII 13 beschriebenen Fischarten (im Gegensatz zu den sog. Uferfischen). An dieser Stelle wechselt Aristoteles das Thema. Er geht über zum Winterschlaf. Die Kapitel Historia animalium VIII 18-27 behandeln Gesundheit und Krankheiten der Tiere.

Die Schlußkapitel von Buch VIII behandeln den Zusammenhang von Habitat und Lebesweise. (Vieles erinnert an Herodots Ausführungen zur Chorologie). Manche Tiere kommen in manchen Gegenden gar nicht vor oder an anderen Orten in anderer Anzahl. Aristoteles nennt Beispiele für Tiere, die an manchen Orten leben, an anderen nicht (Hasen, Wiesel, Maulwürfe). Einige Tiere (wie die libysche Riesenschlange) sind in anderen Regionen signifikant größer. Schlangen- und Skorpiongift ist in Afrika gefährlicher. In Asien leben mehr Löwen als in Europa. Panther leben nur in Asien. Generell sind die wilden Tiere in Asien noch wilder, in Europa aber mutiger, in Afrika vielfältiger geformt. Die Habitate beeinflussen die Verhaltensweisen. Die afrikanischen Tiere sind z.B. wegen des Wassermangels dort aggressiver. Am Schluß des Buchs ist vom Einfluß der Brunst und Trächtigkeit auf das Verhalten und vom Farbwechsel einiger Mollusken die Rede. Die Echtheit von Historia animalium IX ist nicht abschließend geklärt. Sachlich setzt es die Ausführungen von Buch VIII fort. Aristoteles behandelt hier die >intern motivierten Verhaltensweisen. In gewisser Weise lassen sich diese Gedanken als Über- 
legungen als Theorie der Tierintelligenz lesen. Aristoteles' beginnt mit einer Bemerkung zu Charakter und Lernfähigkeit:

Der Charakter der Tiere, die weniger auffällig sind und ein kürzeres Leben haben, ist für die Wahrnehmung weniger offenbar als bei denjenigen Tieren, die länger leben. Die letzteren nämlich besitzen offenbar gewisse natürliche Fähigkeiten, die sich in den Zuständen der Psyche zu erkennen geben, in der Klugheit und Einfalt, dem Mut und der Feigheit, in der Sanftmut und der Bösartigkeit und den übrigen dieser Eigenschaften. Manche Tiere sind auch bis zu einem gewissen Grade des Lernens und der Belehrung fähig, die sie teils voneinander und teils von den Menschen erhalten - und besonders diejenigen, die Gehör besitzen, und zwar nicht diejenigen, die nur Schallunterschiede wahrnehmen, sondern die, die auch Unterschiede in den Sprachzeichen erkennen (HA IX 1, 608a11-21).

In einem knappen Exkurs folgen Gedanken zum geschlechtsspezifischen Charakter der Tiere. Weibchen sind ausnahmslos sanfter, lassen sich leichter zähmen, eher anfassen, sind gelehriger, meist aber (Ausnahme: Löwe, Leopard) feiger, bösartiger, verschlagener, voreiliger und eifriger auf die Ernährung ihrer Jungen bedacht. Männchen sind in der Regel tapferer, heftiger, wilder, offener, weniger hinterlistig und eher hilfsbereit. Aristoteles beginnt die Ausführungen zur Tierintelligenz mit den domestizierten Tieren (Meyer 2017). Unter den Haustieren gelten Schafe, Ziegen und Rinder als besonders einfältig. Pferde schätzt er wegen ihrer großen Liebe zu den Fohlen am meisten. Unter den wilden Säugetieren hebt er die Intelligenz der Hirsche hervor, die sich und ihre Jungen geschickt vor Gefahr und Feinden verstecken ( $H A$ IX 5). Historia animalium IX 7 enthält Notizen zur tierischen Mimesis: »Überhaupt kann man in der Lebensweise der anderen Tiere vielerlei Nachahmungen des menschlichen Lebens beobachten« (HA 612b18 f.). Als Beispiel führt Aristoteles die Schwalben an, die ihre Nester aus einer ähnlichen Verbindung von Lehm und Spreu bauen wie die Menschen. Ein weiteres Indiz ihrer Klugheit ist die auf beide Geschlechter verteilte Brutpflege. Schwerfällige und flugunfähige Vögel (Wachteln, Steinhühner) bauen keine Nester. Sie sind weniger intelligent. Ausführlich würdigt Aristoteles die besondere Klugheit der Kraniche, die Gutmütigkeit der Schwäne und den (ihren Tod ahnenden) Schwanengesang (HA IX 12). Ebenfalls Eichelhäher und Kuckuck sind ausnehmend klug. Auch im Leben der Meerestiere lassen sich »viele technische Kunstgriffe « beobachten. Paradigmatisch ist der Seeteufel, der mit seinen (vor den Augen liegenden) Fäden andere Fische »angelt«. Der Zitterrochen zeigt ein intelligentes Jagdverhalten. Er betäubt die Fische, deren er sich bemächtigen will, mit dem Organ, das er im Maule hat, faßt und verzehrt sie dann. Er verbirgt sich im Sand und faßt die herbeischwimmenden Fische, die beim Herannahen betäubt wurden ( $H A$ IX 37). Von den Mollusken ist die Sepie am raffiniertesten: Sie allein gebraucht Tinte, um sich zu verbergen. Polyp und Kalmare tun dies bloß aus Furcht (ebd.); überhaupt ist der Polyp eher dumm. Unter den Insekten schätzt Aristoteles Ameisen und Bienen wegen ihres Fleißes. Obgleich er detaillierte Beobachtungen zu Bienen (Wabenbau, Honiggewinnung) vorlegt, fehlen hier (wie in den Kapiteln über Wespen und Ameisen) Hinweise 
auf Klugheit. Hervorgehoben werden der Netzbau der Spinnen und ihr intelligentes Jagdverhalten. Summa summarum lassen sich folgende Kriterien für Tierintelligenz angeben (Meyer 2017):

a. Grad der sozialen Organisation, besonders >Arbeitsteilung < (entweder zwischen den Geschlechtern bzw. bei >Schwärmen< die Verteilung von Funktionen auf differente Individuen).

b. Antizipatives Verhalten (Modi des Hortens bzw. Versteckens von Nahrung oder Brut, Trennung von Jagd- und Wohnrevier, Verhalten bei Gefahr).

c. Geschickte Jagdtechniken (Verbergen, Betäuben).

d. Brutpflegeverhalten (Optimum: Männchen und Weibchen arbeitsteilig).

e. Technische Kunstfertigkeit (Nestbau, Netzbau, Reparatur der Spinnennetze, Fleiß).

f. Lernfähigkeit als »Nachahmung« entweder des Menschen oder anderer Tiere (differente Stimmen bei Vögeln, Erkennung von Sprachzeichen).

Insgesamt läßt sich sagen, daß der Mensch überall das Maß für die Fähigkeiten der Tiere abgibt und Aristoteles' Ethologie daher stark anthropozentrische Züge aufweist.

\section{Allgemeine Erklärungen zum Leben}

An vielen Stellen in den zoologischen Schriften des Aristoteles (insb. in der Historia animalium) finden sich Angaben zur Lebensdauer der Tiere. In der Schrift Über die Lebensdauer der Tiere diskutiert Aristoteles die Gründe für Kurz- und Langlebigkeit der Lebewesen (ausführlich: Meyer 2015b).

Säugetiere

\begin{tabular}{l|l} 
Katze & 6 Jahre \\
\hline Ägyptisches Ichneumon & 6 Jahre \\
\hline Ziege & 8 (in Aithiopien 10 bis 11) Jahre \\
\hline Schaf & max. 10-11 (in Aithiopien 12-13), Leithammel 15 Jahre \\
\hline Hirsch & ca. 8 (Kritik mythischer Angaben zur Langlebigkeit) \\
\hline Hund & 12 bis 14 (max. 20) Jahre \\
\hline Schwein & 15 (max. 20) Jahre \\
\hline
\end{tabular}




\begin{tabular}{|c|c|}
\hline Rind & 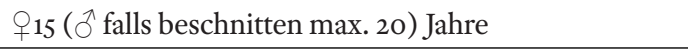 \\
\hline Pferd & 18 bis $20(ð 35 /+40$; max. 75) Jahre \\
\hline Esel & 30 ( + länger) Jahre \\
\hline Maulesel & »lang «, max. 8o Jahre \\
\hline Delphin & viele Jahre, manche 25 bis 30 Jahre \\
\hline Kamel & 30 bis 50 (max. 100) Jahre \\
\hline Elefant & 200 (max. 300) Jahre [basiert auf anonymen Berichten] \\
\hline
\end{tabular}

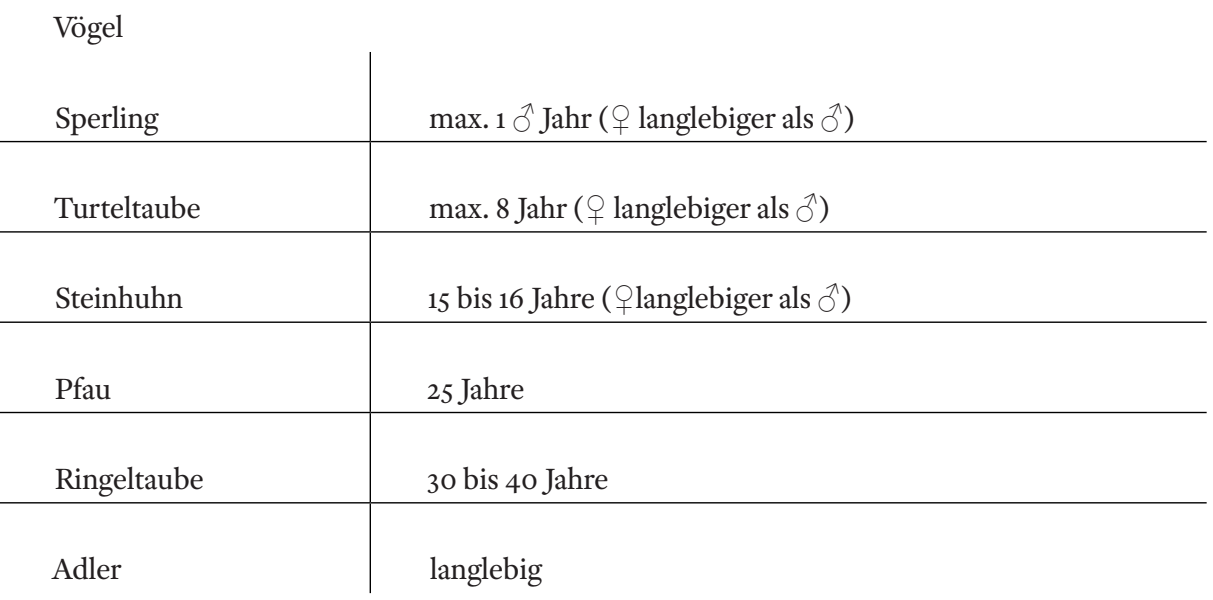

Fische

\begin{tabular}{l|l} 
Thunfisch & 2 Jahre \\
\hline Aal & 7 (max. 8) Jahre
\end{tabular}




\begin{tabular}{l|l} 
Reptilien & \\
Eidechse & 0,5 Jahre \\
\hline Flußkrokodil & lange Lebensdauer
\end{tabular}

Insekten

\begin{tabular}{l|l} 
sog. Ephemeron & 1 Tag \\
\hline Biene & $6(\max .7)$ Jahre (»länger als manche Bluttiere $«)$
\end{tabular}

Mollusken

\begin{tabular}{l|l} 
Mollusken allg. & max. 1 Jahr \\
\hline Polypoden & kurzlebig, max. 2 Jahre \\
\hline Sepie & kurzlebig, max. 2 Jahre \\
\hline Kalmar & max. 2 Jahre
\end{tabular}

Ostrakoderma

\begin{tabular}{l|l} 
Purpurschnecke & 6 Jahre \\
\hline Trompetenschnecken & langes Leben
\end{tabular}

Crustacea

Langusten

alle langlebig 


\section{BIBLIOGRAPHIE}

ALthofF, J., 1997, “Aristoteles' Vorstellung von der Ernährung der Lebewesen“, in: W. Kullmann, S. Föllinger (Hgg.), Aristotelische Biologie. Intentionen, Methoden, Ergebnisse (Akten des Symposions über Aristoteles Biologie vom 24. Bis 28. Juli 1995 in der Werner-Reimers-Stiftung in Bad Homburg), Stuttgart, S. 351-364.

AцtноFF, J., 1992, "Das Konzept der generativen Wärme bei Aristoteles", Hermes 120, S. 181-193.

АстноғF, J., 2006, "Antike Zeugungslehren im Vergleich. Ein Blick auf die Forschungsmethodik des Aristoteles in De generatione animalium", in: J. Althoff, B. Herzhoff, G. Wöhrle (Hgg.), Antike Naturwissenschaft und ihre Rezeption, Bd. 16, Trier, S. 35-50.

Aubert, H., 1862, Die Cephalopoden des Aristoteles, Leipzig.

Aubert, H., Wimmer, F., 1992a, Aristoteles, Thierkunde, Bd. I, Frankfurt am Main [unveränderter Nachdruck der Ausgabe Leipzig 1868].

Aubert, H., Wimmer, F., 1992b, Aristoteles, Thierkunde, Bd. II, , Frankfurt am Main [unveränderter Nachdruck der Ausgabe Leipzig 1868].

BALME, D. M., 1972, Aristotle's De Partibus Animalium I and De Generatione Animalium I (with passages from II 1-3), Oxford.

CAPELLE, W., 1955, "Das Problem der Urzeugung bei Aristoteles und Theophrast und in der Folgezeit", Rheinisches Museum für Philologie 98, S. 150-180.

Cessi, V., 1997, “Anmerkungen zu Aristoteles’ Schrift De sensu“, in: W. Kullmann, S. Föllinger (Hgg.), Aristotelische Biologie. Intentionen, Methoden, Ergebnisse (Akten des Symposions über Aristoteles' Biologie vom 24. Bis 28. Juli 1995 in der Werner-Reimers-Stiftung in Bad Homburg), Stuttgart, S. 325-331.

CharLes, D., 1997, "Aristotle and the Unity and Essence of Biological Kinds", in: W. Kullmann, S. Föllinger (Hgg.), Aristotelische Biologie. Intentionen, Methoden, Ergebnisse. (Akten des Symposions über Aristoteles” Biologie vom 24. Bis 28. Juli 1995 in der Werner-Reimers-Stiftung in Bad Homburg), Stuttgart, S. 63-84.

Cно, D. H., 2003, Ousia und Eidos in der Metaphysik und Biologie des Aristoteles, Stuttgart.

Сно, D. Н., 2010, "Beständigkeit und Veränderlichkeit der Spezies in der Biologie des Aristoteles", in: S. Föllinger (Hg.), Was ist Leben? Aristoteles' Anschaunnen zur Entstehung und Funktionsweise von Leben (Akten der 10. Tagung der Karl und Gertud Abel-Stiftung vom 23. Bis 26. August 2006 in Bamberg), Stuttgart, S. 299-314.

Corcilıus, K., 2008, Streben und Bewegen. Aristoteles' Theorie der animalischen Ortsbewegung, Berlin-New York. DARwin, C.,1882, Letter to William Ogle [February 1882].

DEPEw, D., 2010, "Incidentally Final Causation and Spontaneous Generation in Aristotle's Physics II and Other Texts“, in: S. Föllinger (Hg.), Was ist Leben? Aristoteles' Anschauungen zur Entstehung und Funktionsweise von Leben (Akten der 10. Tagung der Karl und Gertrud Abel-Stiftung vom 23. Bis 26. August 2006 in Bamberg), Stuttgart, S. 285-297.

Dierauer, U., 1977, Tier und Mensch im Denken der Antike. Studien zur Tierpsychologie, Anthropologie und Ethik, Amsterdam.

Drossaart Lulofs, H. J., Poortman, L. J. (ed.), 1989, Nicolaus Damascenus, De Plantis, Amsterdam.

DüRING, I., 1966, Aristoteles. Darstellung und Interpretation seines Denkens, Heidelberg.

Flashar, H., 2010, "Urzeugung und/oder spontane Entstehung", in: S. Föllinger (Hg.), Was ist Leben? Aristoteles' Anschauungen zur Entstehung und Funktionsweise von Leben (Akten der 10. Tagung der Karl und Gertrud Abel-Stiftung vom 23. Bis 26. August 2006 in Bamberg), Stuttgart, S. 331-338. 
FöLlinger, S., 1997, "Die aristotelische Forschung zur Fortpflanzung und zur Geschlechtsbestimmung der Bienen“, in: W. Kullmann, S. Föllinger (Hgg.), Aristotelische Biologie. Intentionen, Methoden, Ergebnisse. (Akten des Symposions über Aristoteles' Biologie vom 24. Bis 28. Juli 1995 in der Werner-Reimers-Stiftung in Bad Homburg), Stuttgart, S. 375-386.

Föllinger, S., 2005, "gonê/Samen“, in: O. Höffe (Hg.), Aristoteles-Lexikon, Stuttgart, S. 237-238.

Frede, M., 1992, “On Aristotle's Conception of the Soul“, in: M. C. Nussbaum, A. O. Rorty (eds.), Essays on Aristotle’s De anima, Oxford, S. 93-107.

Herzhoff, B., 1999, "Das Erwachen des biologischen Denkens bei den Griechen“, in: G. Wöhrle (Hg.), Geschichte der Mathematik und der Naturwissenschaften in der Antike, Bd. 1: Biologie, Stuttgart, S. 13-49.

Herzhoff, B., 2006, "Ist die Schrift »De Plantis« von Aristoteles?", in: J. Althoff, G. Wöhrle, B. Herzhoff (Hgg.), Antike Naturwissenschaft und ihre Rezeption, Bd. 16, Trier, S. 69-108.

Hirschberger, M., 2001, “Aristoteles' Einteilung der Lebewesen in Bluttiere und Nicht-Bluttiere im Lichte der modernen Biologie“, in: J. Althoff, B. Herzhoff, G. Wöhrle (Hgg.), Antike Naturwissenschaft und ihre Rezeption, Bd. 11, Trier, S. 61-71.

KING, R. A., 2004, Aristoteles, De memoria et reminiscentia, in: Aristoteles, Werke in deutscher Übersetzung, H. Flashar (Hg.), Bd. 14.2, Berlin.

KING, R. A., 2005, "sperma/Same/Sperma", in: O. Höffe (Hg.), Aristoteles-Lexikon, Stuttgart, 533-534

KInG, R. A., 2011, "Parva naturalia", in: Ch. Rapp, K. Corcilius (Hgg.), Aristoteles Handbuch. Leben - Werk Wirkung, Stuttgart-Weimar, S. 97-105.

Kollesch, J., 1985, Aristoteles, Über die Bewegung der Lebewesen. Über die Fortbewegung der Lebewesen, in: Aristoteles, Werke in deutscher Übersetzung, H. Flashar (Hg.), Bd. 17.2-3), Berlin.

KraAK, W., 1940, Vogeltrek In De Oudheid In Het Bijzonder Bij Aristoteles, Amsterdam.

Kullmann, W., 1974, Wissenschaft und Methode. Interpretationen zur Aristotelischen Theorie der Naturwissenschaft, Berlin-New York.

Kullmann, W., 1979, Die Teleologie in der aristotelischen Biologie. Aristoteles als Zoologe, Embryologe und Genetiker, Heidelberg.

KullmanN, W., 1997, "Die Voraussetzungen für das Studium der Biologie nach Aristoteles", in: W. Kullmann, S. Föllinger (Hgg.), Aristotelische Biologie. Intentionen, Methoden, Ergebnisse (Akten des Symposions über Aristoteles' Biologie vom 24. Bis 28. Juli 1995 in der Werner-Reimers-Stiftung in Bad Homburg), Stuttgart, S. 43-62.

KullmanN, W., 1998, Aristoteles und die moderne Wissenschaft, Stuttgart.

KullmanN, W., 2000, "Die Beschreibung des Krokodils", in: J. Althoff, B. Herzhoff, G. Wöhrle (hgg.), Antike Naturwissenschaft und ihre Rezeption, Bd. 10, Trier, S. 83-96

KullmanN, W., 2007, Aristoteles, Über die Teile der Lebewesen, in: Aristoteles, Werke in deutscher Übersetzung, H. Flashar (Hg.), Bd. 17.1, Berlin.

Kullmann, W., 2014, Aristoteles als Naturwissenschaftler, Boston-Berlin-München.

LeroI, A. M., 2017, Die Lagune oder wie Aristoteles die Naturwissenschaften erfand, S. Schmidt-Wussow, M. Roth (Übers.), Darmstadt.

LiatsI, M., 2000, Aristoteles, De Generatione Animalium, Buch V. Einleitung und Kommentar, Trier.

Matthews, G. B., 1992, "De anima 2. 2-4 and the Meaning of Life", in: M. C. Nussbaum, A. O. Rorty (eds.), Essays on Aristotle's De Anima, Oxford, S. 185-194.

Maxwell-Stuart, P.-G., 1996, "Theophrastus the traveller”, La Parola del Passato 51, S. 241-267. 
MAYR, E., 2002, Die Entwicklung der biologischen Gedankenwelt. Vielfalt, Evolution, Vererbung, Karin de Sousa Ferreira (Übers), Berlin-Heidelberg-New York.

Meyer, M. F., 2006, "Der Mensch als Maß und Muster. Anthropozentrische Momente in der aristotelischen Biologie“, in: J. Althoff, G. Wöhrle, B. Herzhoff (Hgg.), Antike Naturwissenschaft und ihre Rezeption, Bd. 16, Trier, S. 19-34.

MeYer, M. F., 2008, "Der Wandel des Psyche-Begriffs im frühgriechischen Denken von Homer bis Heraklit", Archiv für Begriffsgeschichte 50, S. 9-28.

Meyer, M. F., 2009, “Demokrit als Biologe“, in: J. Althoff, G. Wöhrle, B. Herzhoff (Hgg.), Antike Naturwissenschaft und ihre Rezeption, Bd. 19, Trier, S. 31-46.

MEYER, M. F., 2011, “Aristoteles über die anatomischen Bedingungen des Sprechens“, in: J. Althoff, S. Föllinger, G. Wöhrle (Hgg.), Antike Naturwissenschaft und ihre Rezeption, Bd. 21, Trier, S. 37-54.

Meyer, M. F., 2012, "Aristoteles über die Psyche als Prinzip und Ursache des Lebens“, Peitho. Examina Antiqua $1(3)$, S. 115-142.

Meyer, M. F., 2013a, “Botanisches Denken von Homer bis Platon“, in: M. Bauks, M. F. Meyer (Hgg.), Zur Kulturgeschichte der Botanik, Trier 2013, S. 107-145.

MeYer, M. F., 2013b, “Aristoteles' Theorie der Atmung in De Respiratione“, in: J. Althoff, S. Föllinger, G. Wöhrle (Hgg.), Antike Naturwissenschaft und ihre Rezeption, Bd. 22, Trier, S. 31-59.

MeYer, M. F., 2015a, Aristoteles und die Geburt der biologischen Wissenschaft, Wiesbaden.

Meyer, M. F., 2015b, “Aristoteles über die Lebensdauer der Tiere und Pflanzen“, in: J. Althoff, S. Föllinger, G. Wöhrle (Hgg.), Antike Naturwissenschaft und ihre Rezeption, Bd. 25, Trier, S. 55-78.

Meyer, M. F., 2016a, “Artform und Individuum in Aristoteles’ Biologie“, in: T. Kirchhoff, K. Köchy (Hgg.), Wünschenswerte Vielheit. Diversität als Kategorie, Befund und Norm, Freiburg-München, S. 59-86.

Meyer, M. F., 2016b, “Organ und Organismus in der aristotelischen Biologie“, in: G. Toepfer, F. Michelini (Hgg.), Organismus. Die Erklärung der Lebendigkeit, Freiburg-München, S. 37-61.

MeYer, M. F., 2016c, "Aristoteles über die menschliche Ontogenese“, in: G. Heinemann, R. Timme (Hgg.), Aristoteles und die heutige Biologie, Freiburg-München, S. 25-52.

Meyer, M. F., 2017, “Aristoteles über die Klugheit der Tiere“, in: J. Althoff, S. Föllinger, G. Wöhrle (Hgg.), Antike Naturwissenschaft und ihre Rezeption, Bd. 27, Trier, S. 141-163.

Meyer, M. F., 2021, "Psyche im griechischen Denken“, in: S. Jöris (Hg.), Die Seele. Zur Genese und Aktualität eines vergessenen Konzepts, Freiburg im Breisgau, S. 21-55 [im Druck].

Oser-Grote, C., 1997, "Das Auge und der Sehvorgang nach Aristoteles und der hippokratischen Schrift De carnibus“, in: W. Kullmann, S. Föllinger (Hgg.), Aristotelische Biologie. Intentionen, Methoden, Ergebnisse. (Akten des Symposiums über Aristoteles' Biologie vom 24. Bis 28. Juli 1995 in der Werner-Reimers-Stiftung in Bad Homburg), Stuttgart, S. 333-349.

Oser-Grote, C., 2004, Aristoteles und das Corpus Hippocraticum. Die Anatomie und Physiologie des Menschen, Stuttgart.

Polansky, R., 2007, Aristotle's De anima, Cambridge.

REPICI, L., 2009, "Il De plantis pseudo-aristotelico nella tradizione antica e médiévale“, in: A. P. Bagliani (Hg.), Le monde végétal. Médecine, botanique, symbolique, Fierenze, S. 77-94.

SChARFEnBERG, L. N., 2001, Die Cephalopoden des Aristoteles im Lichte der modernen Biologie, Trier. 
Sснмiтт, A., 1997, "Verhaltensforschung als Psychologie. Aristoteles zum Verhältnis von Mensch und Tier", in: W. Kullmann, S. Föllinger (Hgg.), Aristotelische Biologie. Intentionen, Methoden, Ergebnisse. (Akten des Symposions über Aristoteles' Biologie vom 24. Bis 28. Juli 1995 in der Werner-Reimers-Stiftung in Bad Homburg), Stuttgart, S. 259-286.

SEIDL, H., 1968, Das Verhältnis der causa efficiens zur causa finalis in Aristoteles' Schrift $\gg$ De generatione animalium «, München.

STrohbach, N., 2008, "Was heißt es, eine ARCHĒ in sich zu haben?"“, in: K. Corcilius, Ch. Rapp (Hgg.), Beiträge zur Aristotelischen Handlungstheorie (Akten der 8. Tagung der Karl und Gertrud Abel-Stiftung vom 08.-11. Juli 2004 in Blankensee), Stuttgart, S. 65-81.

StÜCKelberger, A., 1998, "Vom anatomischen Atlas des Aristoteles zum geographischen Atlas des Ptolemaios. Beobachtungen zu wissenschaftlichen Bilddokumentationen“, in: W. Kullmann, J. Althoff, M. Asper (Hgg.), Gattungen wissenschaftlicher Literatur in der Antike, Tübingen, S. 287-307.

VAN DER EIJK, Ph., 1994, Aristoteles, Parva naturalia III : De insomniis. De divinatione per somnum, in: Aristoteles, Werke in deutscher Übersetzung, H. Flashar (Hg.), Bd. 14.3, Berlin.

VAN DER EIJK, Ph., 2005, Medicine and philosophy in classical antiquity, Doctors and Philosophers on Nature, Soul, Health and Disease, Cambridge.

WöHRLE, G., 1985, Theophrasts Methode in seinen botanischen Schriften, Amsterdam.

WöHrLE, G., 1997, "Aristoteles als Botaniker“, in: W. Kullmann, S. Föllinger (Hgg.), Aristotelische Biologie. Intentionen, Methoden, Ergebnisse. (Akten des Symposions über Aristoteles' Biologie vom 24. Bis 28. Juli 1995 in der Werner-Reimers-Stiftung in Bad Homburg), Stuttgart, S. 387-396.

ZELLER, E., 1862, Die Philosophie der Griechen in ihrer geschichtlichen Entwicklung, Zweiter Theil. Zweite Abtheilung: Aristoteles und die Peripatetiker, 2. Auflage, Tübingen.

ZIERLEIN, S., 2013, Aristoteles, Historia animalium. Buch I und II, in: Aristoteles, Werke in deutscher Übersetzung, Ch. Rapp (Hg.), Bd. 16, Berlin. 
MARTIN F. MEYER

/ University of Koblenz /

mmeyer@uni-koblenz.de

\section{Aristotelian Biology. A Synopsis}

In no field of knowledge did Aristotle leave more writings than in biology. He conducted research for longer and more intensively in zoology than in any other field. In these writings he mentions a good 550 animal and 60 plant species. While this includes the internal anatomy of around 110 animals, he dissected 60 species himself. The present contribution deals with the epistemic motifs and the meaning of Aristotelian biology in the context of his scientific curriculum. It is thus demonstrated that in De anima Aristotle's actual explanations are preceded by an investigation of the principles, which aims to differentiate living objects from inanimate ones, and to develop a method of explanation based on the species-specific vital functions of living beings. This article provides an overview of the four main disciplines of Aristotelian biology: comparative anatomy, physiology, genetics and behavioral research. The text offers tabular overviews of the animals and plants dealt with by Aristotle.

science, natural science, biology, life, vital functions, zoology, botany, anatomy, physiology, genetics, behavioral research 
